\title{
Solar EUV spectroscopic observations with SOHO/CDS
}

\section{An in-flight calibration study}

\author{
G. Del Zanna ${ }^{1, \star}$, B. J. I. Bromage ${ }^{2}$, E. Landi ${ }^{3, \star \star}$, and M. Landini ${ }^{4}$ \\ 1 Department of Applied Mathematics and Theoretical Physics, University of Cambridge, Cambridge, UK \\ 2 Centre for Astrophysics, University of Central Lancashire, Preston, UK \\ 3 Naval Research Laboratory, Washington DC, USA \\ 4 Dipartimento di Astronomia e Scienza dello Spazio, Università di Firenze, Firenze, Italy
}

Received 2 May 2000 / Accepted 29 August 2001

\begin{abstract}
An in-flight calibration study of the Coronal Diagnostic Spectrometer (CDS) instrument on board SOHO (Solar and Heliospheric Observatory) is presented. The relative intensity calibration of CDS is a fundamental requirement for deriving important physical parameters of the solar transition region and corona from the observations. This comprehensive study provides the first complete in-flight relative calibration of all nine CDS channels, first and second order. This has been achieved with the use of a spectroscopic calibration method, mainly based on the comparison between observed line ratios and theoretical predictions provided by the CHIANTI atomic database. The calibration method has been applied to a large number of observations (on-disc, off-limb, quiet sun, active region), to enable the use of a wide range of spectral lines from low to high temperatures of formation. The results are compared to the pre-launch calibration and other post-launch studies. Significant differences with the ground calibration results are found, while there is good agreement with the post-launch studies, based on rocket flights. It is also shown that the relative calibration has not significantly changed over a long period of time, thus confirming the excellent stability of the CDS instrument.
\end{abstract}

Key words. Sun: corona - techniques: spectroscopic

\section{Introduction}

The NASA/ESA Solar and Heliospheric Observatory (SOHO) was launched successfully in December 1995 and has since produced a wealth of observations of the Sun. The Coronal Diagnostic Spectrometer (CDS) observes the solar corona in the Extreme-Ultraviolet (EUV), covering a wide wavelength range (150-780 $)$, with six detectors. In June 1998 the SOHO spacecraft lost power and contact with Earth. After various attempts, the spacecraft was successfully recovered in September 1998. However, since recovery, the SOHO instruments (and in particular CDS) have continued to provide observations at the same rate as before.

A description of the CDS instrument can be found in Harrison et al. (1995). Here, a summary of the

\footnotetext{
Send offprint requests to: G. Del Zanna,

e-mail: G.Del-Zanna@damtp.cam.ac.uk

* Previous address: Centre for Astrophysics, University of Central Lancashire, Preston, UK

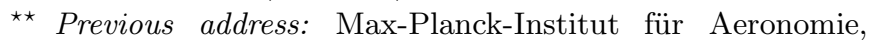
Katlenburg-Lindau, Germany
}

characteristics relevant for this paper is given in Sect. 2 . More details can be found in Del Zanna (1999). The CDS instrument offers the opportunity of on-disc and off-limb observations of many emission lines formed over a large temperature range, emitted from the chromosphere to the solar corona. This allows a large number of EUV plasma diagnostic techniques to be used for investigating the characteristics of the solar atmosphere (see, e.g., Mason et al. 1997; Young \& Mason 1997; Landi \& Landini 1998a; Del Zanna \& Bromage 1999a; Del Zanna \& Bromage 1999b; Mason et al. 1999). In particular, densities, temperatures and element abundances of the solar transition region and corona can be determined with CDS to a good accuracy. However, any uncertainty in the relative intensity calibration affects the determination of these parameters. Particularly if the emission lines are not close in wavelength or are seen in different detectors.

The relative intensity calibration between and along the CDS detectors is therefore a fundamental requirement for any scientific use of the CDS observations. Obtaining such information is not straightforward in the extreme 
ultraviolet spectral range. Previous measurements of the CDS calibration suffered from large uncertainties or limitations (see Sect. 3) and a new in-flight study was needed.

The aim of the present work is to derive a comprehensive in-flight relative calibration of all CDS channels, first and second order. The relative calibration should provide overall agreement between observations and theory, with an uncertainty of the order of the uncertainties in the atomic data and the observations (20-30\%). This will ensure that the physical quantities estimated using spectral diagnostic techniques applied to lines observed in any CDS channel are as accurately determined as possible.

The CDS absolute intensity calibration is not derived here. Any change in the absolute values does not affect the determination of the physical parameters (except for absolute emission measures). What is derived here is the intensity calibration as a function of wavelength for each channel (the internal calibration), and the relative calibration between the various channels and orders (the crosscalibration).

The core of the calibration method adopted here is based on a spectroscopic method, described in Sect. 4 . Section 4 also discusses the applicability of the method to the available CDS observations and why new observations were needed. Section 5 describes how the new CDS observations were planned and analysed.

The calibration process can be summarised in four steps.

- The first step (Sect. 6) comprises the selection of the most suitable observations, that is where the effects of solar variability are negligible.

- The second step (Sect. 7) consists in the direct cross-calibration between spectrally-overlapping regions. This cross-calibration is straightforward, does not depend on the atomic data, and allows a direct check of the relative calibration between the overlapping channels and its stability over the years.

- The third step (Sect. 8) is the selection of the most suitable line ratios to be used for the calibration and the most appropriate observations to be used.

- The fourth step consists in the application of the spectroscopic method.

The results, in terms of relative calibration between all the CDS channels, in first and second order, are presented and compared with previous calibration studies in Sect. 9, while Sect. 10 draws the conclusions. Details on the lines used for the calibration are given in the Appendix.

\section{Overview of the instrument}

The CDS instrument consists of a Wolter-Schwarzschild type II grazing incidence telescope, a scan mirror, a set of different slits, and two spectrometers that do not observe simultaneously: the normal incidence spectrometer (NIS) and the grazing incidence spectrometer (GIS).

The NIS is composed of two normal incidence gratings that disperse the light into the NIS detector, mainly composed of a microchannel plate (MCP) and a charge coupled device (CCD). The gratings are slightly tilted, in order to produce two wave-bands (NIS 1: 308-379 $\AA$ and NIS 2: $513-633 \AA$ ). The spectral resolution in terms of fullwidth at half-maximum (FWHM) is $\simeq 0.35 \AA$ for NIS 1 and $\simeq 0.5 \AA$ for NIS 2 . The NIS can provide monochromatic images of the field of view by moving a scan mirror to produce contiguous images of one of the long slits (a "raster"). Telemetry constraints usually make it necessary to reduce the amount of information recorded on board, before the data are telemetred to the ground. NIS spectra contain a number of effects that limit their usability. For example, NIS spectra have a wavelength-dependent scattered light ("background") component, that is mostly concentrated in the network areas, and which disappears in off-limb observations. The NIS 1 spectral region, where CDS has many potential diagnostic lines, is the most affected. The uncertainty in estimating this background component results in an uncertainty in the line intensity evaluation. The presence of cosmic ray hits strongly constrains the NIS observations, as exposure times cannot be too long, otherwise cosmic rays prohibit the use of large fractions of the spectra. Some geometrical distortions of the NIS spectra are also present (see, e.g., Haugan 1999), which complicate the observations and the data analysis.

The grazing incidence spectrometer (GIS) uses a grazing incidence spherical grating that disperses the incident light to four microchannel plate detectors placed along the Rowland Circle (GIS 1: 151-221 A, GIS 2: 256-341 $\AA$, GIS 3: 393-492 $\AA$ and GIS 4: 659-785 $\AA$ ). The spectral resolution of the GIS detectors is about $0.5 \AA$. The GIS is astigmatic, focusing the image of the slit along the direction of dispersion but not perpendicular to it. Images of the Sun are obtained using a pinhole slit and combining movements of the slit and of the scan mirror. The GIS detectors are SPAN (spiral anode) micro-channel plates (Breeveld 1996). A set of parameters, identified by a unique number (GSET_ID), is used on board the spacecraft to convert the signal produced by each photon event to create 2048 spectral data points.

An advantage of the GIS over the NIS is that it is possible to obtain spectra with a high cadence. In fact, with normal CDS telemetry the four GIS spectra can be down-loaded in about $13 \mathrm{~s}$. Telemetry is therefore not a limitation for GIS.

However, there is an instrumental effect that limits the scientific use of the GIS spectra. In some regions of the GIS spectra, a portion of the counts belonging to a line can be shifted into one or two different regions of the telemetered spectrum. This gives rise to spurious spectral lines if they fall in a spectral region void of lines, or adds extra intensity to already existing spectral lines. Henceforth we will refer to a spectral line whose intensity is enhanced in this way as a ghosted (or contaminated) line, while the lines whose counts have been partially shifted toward other regions of the spectrum will be referred to as ghosting (or parent) lines. The counts shifted by this effect will be called ghosts, while the process will be called simply ghosting. 
The GIS spectra have to be deghosted (or reconstructed), before any scientific use can be made of them. More details can be found in Breeveld (1996), Landi et al. (1999a), and Del Zanna (1999).

Second order lines have been observed in NIS 2, GIS 3 and GIS 4. The GIS and NIS sensitivities are such that in many cases second order lines are either prominent features in the spectra, or strongly blend with first order lines. An accurate knowledge of the second order sensitivities is therefore required, for a correct interpretation of the spectra.

The presence of MCPs in both the NIS and GIS detectors causes a decrease of their sensitivity with time, due to the exposure to solar radiation. For the NIS, this results in a depression at the core of the lines (the socalled burn-in of the lines), that is non-negligible for the brightest lines. This effect in NIS spectra has been monitored since launch using wide slit observations, and can be corrected for by reconstructing the line profiles with good accuracy (typically a few percent), using standard CDS software. For the GIS, the overall gain depressions for each channel were corrected at the beginning of the mission by increasing the voltage of the MCPs. However, no clear indications of burn-in of the GIS lines has been found (with the exception of the He II $304 \AA$ line). On top of the local (in time and spatial) gain depressions for both NIS and GIS detectors, also a long-term gain depression was expected. A gain depression across the GIS detectors was particularly evident during the first few months of operations, but since then appears to have stabilised. The goal of the instrument calibration is also to monitor and characterise these long-term effects.

The CDS performance changed in many respects after SOHO was recovered. The details of these changes, some of which are still under investigation, are beyond the scope of this paper. The main differences that are relevant to this paper are: 1) an offset of about $15^{\prime \prime}$ between the NIS and the GIS observations; 2) the NIS spectra present broadened and asymmetrical (for NIS 2) line profiles, that in particular for NIS 1 strongly reduce the spectral resolution. The GIS spectra did not suffer any major changes, except for GIS 2 which was switched off for a long period of time.

\section{Background to the CDS calibration}

A pre-launch calibration was performed by Bromage et al. (1996), producing an absolute calibration for both NIS and GIS, in first order. These measurements provided an initial measure of the mean sensitivity of each detector and a basis for the absolute calibration. Additional information was provided by the theoretical wavelength dependence of the sensitivities. C. D. Pike and H. E. Mason used in-flight measurements to perform a preliminary correction to the Bromage et al. (1996) NIS values, and to provide new NIS sensitivities. These were stored for public use in the CDS data analysis software until
December 1998, and will be referred here as the "first NIS standard calibration".

The first NIS standard calibration was later found to require corrections by various authors (see, e.g., Landi et al. 1997), the main one being the need to increase the NIS 1 sensitivity, relative to NIS 2, by some factor. Landi et al. (1997) proposed a factor between 2.5 and 5. The large uncertainty was mainly due to the lack of lines emitted by the same ions visible in the two detectors.

An in-flight cross-calibration between the NIS and a rocket flight launched in May 1997 has been presented by Brekke et al. (2000). This study has produced absolute values of the in-flight NIS sensitivities, for both NIS 1 and NIS 2. For NIS 2, it was possible to estimate a coarse wavelength-dependence. For NIS 1, only one reliable measurement at one wavelength was possible.

A revised pre-launch calibration for both GIS and NIS in first order has been presented by Lang et al. (1999, 2000). Only measurements at a few wavelengths were performed on the ground. One measurement for the NIS 2 in second order was also provided, at $608 \AA$. This revised pre-launch calibration suggests a different NIS calibration, both in absolute and relative (between NIS 1 and NIS 2) terms. The revised value of the absolute sensitivity at $584 \AA$ (NIS 2) is the same as the in-flight value obtained by Brekke et al. (2000), while at the other wavelengths the two calibrations are not in good agreement.

A "second NIS standard calibration", based on the results of Brekke et al. (2000) and Lang et al. (2000), was therefore implemented in the CDS data analysis software in December 1998.

Recently, since March 2000, a "third NIS standard calibration" has been implemented in the standard CDS software. Only the NIS 1 sensitivity was changed (see below for details), based on a preliminary CDS / SERTS-97 cross-calibration, performed by Thomas et al. (1999).

Regarding GIS, a preliminary in-flight study of the relative intensity calibration of the GIS detectors was performed by Landi et al. (1999a). In this study, a quiet sun and an active region on-disc spectrum were used to check the relative averaged values of the pre-launch (Bromage et al. 1996) calibration, assuming constant sensitivity across each detector. An overall agreement (within the uncertainties) was found. Regarding second order sensitivities, no pre-launch measurements were available. A preliminary assessment of the GIS 3, 4 sensitivities in second order was also given by Landi et al. (1999a). However, these results were limited by the fact that only GIS observations were used, and therefore most of this second order calibration depended solely on theoretical predictions.

In summary, the pre-launch measurements only provided sensitivities for some of the channels, and only at a few wavelengths. Moreover, these sensitivities may not be directly applicable to the in-flight observations, for several reasons. First, the pre-launch calibration was based on spectra taken on the ground nearly two years before launch (in March 1994), and changes in the 
sensitivities could have taken place. Then, during the prelaunch calibration, the detectors were illuminated by a narrow-beam calibration source, while in-flight they are fully-illuminated by the Sun. Finally, variations in time could be expected, due to gain depressions in the MCPs, or related to a decreased reflectivity of the telescope's mirrors, the scan mirror and the gratings, which can be due to deposits. The previous in-flight studies provided estimates of the relative sensitivities for either the NIS or the GIS channels, but not both together.

It is therefore clear that an in-flight calibration of all the CDS detectors was necessary, together with an assessment of any in-flight variations with time. In the absence of any suitable on-board calibration source, the only possibility is to use the solar emission as the calibration source, and to apply a spectroscopic method.

\section{The spectroscopic method}

The idea is to choose only those groups of "calibration" lines whose ratios are expected to be independent of the observed source. The first ratios to examine are branching ratios (i.e. ratios of lines that share a common upper level). These intensity ratios depend only on the radiative transition probabilities, which are known to $\sim 10 \%$ accuracy. Next, ratios of density-insensitive lines are used. The procedure is then to apply the comparison to all the available ions. When agreement is found between the results obtained by the use of many lines of different ions, the relative calibration between the various channels is obtained. The procedure can then be applied to observations taken at different times, to study any changes of the sensitivities.

This method has already been applied a few times in the past. Neupert \& Kastner (1983) used this method for an in-flight calibration of the OSO V and OSO VII EUV spectrometers, in the 150-400 A range. Recently, the method was successfully applied (Brosius et al. 1998a; Brosius et al. 1998b) to calibrate the Solar EUV Rocket Telescope and Spectrometer observations in 1995 (SERTS$95)$, using averaged active region and quiet sun spectra, and CHIANTI (version 1.01, except for Fe XIV). The same method was also applied by Young et al. (1998) to check the calibration of the SERTS-89 active region spectrum (Thomas \& Neupert 1994).

In order to make full use of this diagnostic method for calibration purposes, given the uncertainties in the atomic data and the observed intensities, it is essential to use as many lines emitted by the same ion as possible. It is also important to use as many ions as possible, in order to reduce the possibility that incorrect atomic data for one single ion would alone affect theresults.

Note that uncertainties in the atomic data are intrinsically difficult to estimate, but typically are $10-30 \%$. In any case, any effects that large errors on atomic data could have on the calibration have been reduced as much as possible. In fact, only calibration ratios that have shown excellent agreement (within 10\%) between previous solar observations and predictions based on atomic theory have been used in most of the cases. There is no reason why the calibration ratios observed by the CDS should differ from the same ratios observed by other instruments, if the same atomic data are used. In this respect, the results on comparisons between CHIANTI predictions and SERTS observations (Young et al. 1998; Del Zanna 1999; Brosius et al. 1998b) have been taken into account here.

Before describing how the calibration method was applied, it is first necessary to provide some definitions. The intensity $I\left(\lambda_{i j}\right)\left[\mathrm{ergs} \mathrm{cm}^{-2} \mathrm{~s}^{-1} \mathrm{sr}^{-1}\right.$ ] of an optically thin spectral line of wavelength $\lambda_{i j}$ can be written in the form:

$I\left(\lambda_{i j}\right)=\int_{h} A b(Z) C\left(N_{\mathrm{e}}, T, \lambda_{i j}\right) N_{\mathrm{e}} N_{\mathrm{H}} \mathrm{d} h$

where the contribution function $C\left(N_{\mathrm{e}}, T, \lambda_{i j}\right)$ of each line contains all the relevant atomic physics parameters and for most of the transitions has a narrow peak in temperature, and therefore effectively confines the emission to a limited temperature range. $A b(Z)$ is the element abundance relative to hydrogen. $N_{\mathrm{H}}, N_{\mathrm{e}}\left(\mathrm{cm}^{-3}\right)$ are the hydrogen and electron number densities. If a unique relationship exists between $N_{\mathrm{e}}(h)$ and $T(h)$, and the abundance of the element $A b(Z)$ is constant over the line of sight, the intensity can be rewritten:

$I\left(\lambda_{i j}\right)=A b(Z) \int_{T} C\left(T, \lambda_{i j}, N_{\mathrm{e}}\right) D E M(T) \mathrm{d} T$

where we have defined the differential emission measure $\operatorname{DEM}(T)=N_{\mathrm{e}} N_{\mathrm{H}} \mathrm{d} h / \mathrm{d} T$. The $D E M$ gives an indication of the amount of emitting plasma along the line of sight with a temperature between $T$ and $T+\mathrm{d} T$. Here, the $D E M$ analysis was performed using a modified version of the Arcetri inversion code (Monsignori Fossi \& Landini 1991).

The best available atomic data, stored in the CHIANTI database and Arcetri Spectral Code, have been used. The CHIANTI atomic database (Dere et al. 1997) is the result of an international collaboration (USA, Italy, UK) to provide a comprehensive dataset for ions of astrophysical interest from X-ray to ultraviolet wavelengths. CHIANTI v.2 (Landi et al. 1999b) has been used for the present analysis. In addition, recent Fe XIV calculations (Storey et al. 2000) have been used here. Note that the new Fe XIV data produce results that are significantly in better agreement with solar observations. In the last few years, new calculations for Fe XII have also been produced (see Binello et al. 2001 and references therein). Fe XII lines have been used here for the calibration of the NIS 1 and GIS 2 detectors (see below). If these new data are used, the NIS 1 calibration would be the same, while the relative sensitivity of GIS 1 would increase at $195 \AA$ by $20 \%$. Recently, some of the CHIANTI atomic data of the ions of the Be-like sequence (Mg IX, O V and Si XI in particular) were found to require corrections. For Mg IX, calculations of $A$-values by P. R. Young (1999, priv. comm.) have been used here. These data are now available in version 3 of the CHIANTI database (Dere et al. 2001). 
The CHIANTI version 2 data for O V and Si XI used here had collisional data calculated by Zhang \& Sampson (1992). If the R-Matrix calculations of Berrington et al. (1985) are used, improvements regarding some transitions are found. However, the use of the Berrington et al. (1985) data does not affect the lines used here for the CDS calibration. The differences in the $\mathrm{O} \mathrm{V}$ line intensities used here for the cross-calibration of NIS 2 and GIS 4 (see below) are negligible, of the order of a few \%. Similarly, the ratio between the $2 \mathrm{~s}^{2}{ }^{1} \mathrm{~S}_{0}-2 \mathrm{~s} 2 \mathrm{p}{ }^{3} \mathrm{P}_{1}(580.91 \AA)$ and the resonance $2 \mathrm{~s}^{2}{ }^{1} \mathrm{~S}_{0}-2 \mathrm{~s} 2 \mathrm{p}{ }^{1} \mathrm{P}_{1}$ line $(303.32 \AA)$ is not substantially affected (a variation of about $10 \%$ ). On the other hand, the $2 \mathrm{~s} 2 \mathrm{p}{ }^{1} \mathrm{P}_{1}-2 \mathrm{p}^{2}{ }^{1} \mathrm{D}_{2} 604.15 \AA$ line has been under-estimated by a factor of about 2 here. For those ions that are not present in the CHIANTI database, the Arcetri Spectral Code (see, e.g. Landi \& Landini 1998b) was used. The contribution functions have been calculated at constant density or pressure, assuming the collisional ionization equilibrium of Arnaud \& Rothenflug (1985).

The adopted calibration method was applied as follows. A first trial calibration was applied to all the observed lines. This was accomplished with the representation of the absolute first and second order sensitivities (see Sect. 5.2) by a spline function for each CDS channel. The relative values of the sensitivities between the overlapping regions (see Sect. 7) were directly derived from the observations. Then, a $D E M$ analysis was performed, fixing the element abundances. Once a $D E M$ distribution was found, the theoretical intensities of the lines were computed and compared to the observed ones. This was done to ensure proper identification of the calibration lines and assess blending, an important issue, given the moderate CDS spectral resolution. Also, to take into account any temperature effects in temperature-sensitive calibration line ratios. It should be mentioned that the determination of the $D E M$ distribution is an ill-posed problem (see, e.g. Craig \& Brown 1986; McIntosh 2000 and references therein) where exact solutions are not unique. However, we are confident that the adopted procedure is appropiate for the present spectroscopic method.

Finally, all the branching ratios and ratios between density-insensitive lines were considered, comparing observed and calculated values. This was done for all the ions and different observations analysed. When discrepancies were found, the calibration was changed and a new iteration started. Note that if only density- and temperatureinsensitive ratios are used, the results are strictly independent of the adopted densities, the ionization equilibrium or $D E M$ distributions.

\subsection{Applicability of the calibration method to the available CDS observations}

Considerable effort was exerted in analysing available CDS observations, to see if the adopted calibration method could be applied to those data. In particular, analyses of spectra of different solar regions (e.g. on-disc and off-limb spectra, as well as quiet sun and active regions) were performed for the following reasons:

1. To assess line identification and blending. The CDS spectral range includes many hundreds of lines, seen in first and second order. Many of these lines are blended, given the moderate CDS spectral resolution. Moreover, blending varies depending on which solar regions is observed. A complete discussion of the line identification issue is beyond the scope of this paper, and will be reported in Del Zanna et al. (2001, hereafter referred to as Paper II);

2. To assess which good calibration ratios should be used. It is important to have a confirmation from the observations which calibration ratios are constant, independently of the observed source;

3. To assess ghosting in the GIS spectra, and which GIS lines can be used for the calibration. For example, a ghost will disappear if its parent line does, as lowtemperature lines do in off-limb observations. In other cases it is difficult to assess the presence of a ghost because it contaminates a "true" line, and it is only when this line is not emitted by the observed solar region, that the observed "line" can be identified as a "pure ghost";

4. To assess the importance of the "background" intensity in the NIS and GIS spectra. In the NIS spectra this component is mainly due to scattered light and disappears in off-limb observations. In GIS spectra, the main background component is due to true $\mathrm{He}$ and $\mathrm{H}$ continua, and so it also disappears off-limb.

After this detailed survey, all the available CDS observations were found to be unsuitable for a complete application of the spectroscopic method. This was for several different reasons (e.g. low signal to noise $(S / N)$, temporal variability of the solar region observed, lack of sufficient spatial overlapping between the NIS and the GIS rasters, due to solar rotation), but the main reason was that fullwavelength NIS and GIS spectra were not telemetered. Many full-wavelength GIS and NIS observations were however used for the separate NIS and GIS internal calibrations, and are listed in the following section. However, only a few reliable calibration line ratios are observed in each spectrometer. The worst case is represented by the NIS, where there are only a few lines (weak and blended) that can be used for the cross-calibration between NIS 1 and NIS 2, as already pointed out by Landi et al. (1997).

The spectroscopic calibration method can therefore be applied to cross-calibrate all the CDS channels only if NIS and GIS observations are considered together.

\section{Observations and data analysis}

Since NIS and GIS detectors cannot be used simultaneously, new near-simultaneous NIS-GIS observing sequences (CDS "studies") had to be carefully planned, taking into account the instrument characteristics and 
various issues that are important when the Sun is used as a calibration source:

- Temporal variability. To reduce the effects of the solar variability, diffuse quiet regions have been chosen. A series of alternate NIS and GIS rasters would then reveal if intensity variations have taken place (see Fig. 1);

- Raster sizes. The raster sizes have been chosen small enough to keep the total duration of the observation as short as possible, but large enough to allow a check on the relative NIS-GIS pointing;

- The location and the spatial variability. In on-disc observations, it is important to be able to cross-correlate the GIS and NIS data for co-alignment, in order to correct for the solar rotation (about $10^{\prime \prime}$ in an hour at the Sun centre), and to compare intensities of the lines that are emitted by the same spatial regions. Small rasters that have short total duration, and for which the effects of the solar rotation are negligible, were therefore designed;

- Good signal. To get higher signal while keeping the time between the NIS and GIS exposures as short as possible, the wider $\left(4^{\prime \prime}\right)$ slits have been chosen. This advantage is not gained at the expense of spectral resolution (the $2^{\prime \prime}$ and $4^{\prime \prime}$ slits produce spectra with the same spectral resolution).

A considerable number of calibration observations have been planned, performed and analysed. They can be broadly subdivided into observations near disc centre and observations near the solar limb. None of the near disc centre observations were found to be fully usable, mainly because of the high variability in all the transition region lines, predominantly located at the supergranular network boundaries.

In contrast, the NIS-GIS sequences performed near the solar limb were found to be suitable for both the internal NIS and GIS calibration, and for the cross-calibration of all the CDS detectors. In the observations near the solar limb, the effects of the solar rotation are reduced by the large line-of-sight, which also produces a much higher signal. This allows high $S / N$ to be achieved by spatially averaging over small areas. The observations near the limb also provide at the same time on-disc and off-limb spectra. The off-limb observations have been invaluable because the scattered light (mostly present in NIS 1) and the continuum (mostly present in GIS 3, 4) disappear off-limb, leaving much cleaner spectra.

The NIS studies that were developed for the observations at the limb are small rasters of full-wavelength spectra using the $4^{\prime \prime} \times 240^{\prime \prime}$ slit. The CDS study name is ARC_CALN/v3, covering an area of $32^{\prime \prime} \times 70^{\prime \prime}$. After correcting for the slant in the spectra, the field of view (FOV) is reduced to $32^{\prime \prime}$ in $X$ and about $40^{\prime \prime}$ in $Y$. With an exposure time of $45 \mathrm{~s}$, it takes less than 13 min to raster the whole area. The GIS observation used the CDS study DEM_GIS/v2, where the $4^{\prime \prime} \times 4^{\prime \prime}$ slit is rastered in order to cover a $24^{\prime \prime} \times 24^{\prime \prime}$ region with 36 spatial positions. The total duration is also just under $13 \mathrm{~min}$, with a $20 \mathrm{~s}$
Table 1. The observations used for the CDS instrument calibration.

\begin{tabular}{lll}
\hline Files (from-to) & Date and time (UT) & CDS studies \\
\hline s7772r00-s7782r00 & $1997 / 05 / 04$ 18:41-21:07 & DEM_GIS, ARC_CALN \\
s7783r00-s7792r00 & $1997 / 05 / 04 ~ 21: 21-23: 33$ & ARC_CALN,DEM_GIS \\
s8229r00-s8238r00 & $1997 / 06 / 20$ 07:08-09:18 & DEM_GIS, ARC_CALN \\
s9298r00-s9307r00 & $1997 / 10 / 0113: 50-16: 01$ & DEM_GIS, ARC_CALN \\
s9454r00-s9463r00 & $1997 / 10 / 1611: 22-13: 06$ & DEM_GIS, ARC_CALN \\
\hline s4554r00 & $1996 / 09 / 02 ~ 22: 42$ & SPECT_1/v1 \\
s8257r00 & $1997 / 06 / 2221: 53$ & NISAT_S/v2 \\
s9253r00 & $1997 / 09 / 2518: 01$ & NISAT_S/v2 \\
s9487r00 & $1997 / 10 / 1906: 41$ & NISAT_S/v2 \\
s10566r00 & $1998 / 03 / 0809: 06$ & UCLAN_N2 \\
\hline s16154-59r00 & $1999 / 05 / 2307: 36-08: 51$ & DEM_GIS, ARC_CALN \\
\hline
\end{tabular}

exposure time. The ARC_CALN and DEM_GIS were repeated in succession about five times, without changing the pointing, at the limb, for a total duration of about 2 hours.

The observations analysed to check the CDS calibration span the years from 1996 to 2000. Table 1 lists only the observations analysed in detail and actually used for the calibration. Table 1 lists three groups of observations: 1) the NIS-GIS sequences performed at the west limb in 1997; These observations have been successful in terms of pointing, telemetry, good signal, and small temporal variability;

2) the separate NIS and GIS full spectra, used for the internal NIS and GIS calibration. These include: a) an on-disc active region spectral atlas (NISAT study) on the 25th September 1997; b) two quiet sun spectral atlases (NISAT studies) at sun centre, on 22nd June 1997 and 19th October 1997; c) an off-limb active region NIS spectrum, kindly provided by S. Parenti, and taken on the 8th March 1998; d) a GIS active region observation, the same one used by Landi et al. (1999a) for the GIS-only calibration;

3) one set of NIS-GIS sequences performed in 1999, after SOHO recovery. These observations were on the disc, close to the south limb.

Only a few "typical" results have been selected for presentation here as examples from this very large dataset.

\subsection{Data analysis}

Various standard corrections were applied to the raw NIS data. These include: de-biasing, flat-fielding, corrections for the burn-in of a few lines, nonlinear corrections (using the CDS routine VDS_CALIB); cosmic ray removal (using the CDS routine CDS_CLEAN_IMAGE together with XCDS_COSMIC, and visual inspection of all the spectra); and correction for the slant of the spectra (using the CDS routine VDS_ROTATE). All the corrections applied have a small error associated with them. For more details see Del Zanna (1999).

A few corrections were applied to the GIS data, as described in Landi et al. (1999a). These corrections are also small, and do not usually exceed $1 \%$. The main effects to 
Table 2. Sample of the ghosting reconstruction applied to the GIS off-limb spectra of May 4th, 1997. The averaged positions of the ghost and parent (i.e. ghosting) lines are indicated in Cols. 1 and 3. The ratio of the intensity of the ghost vs. that of the ghosting line (in counts) is presented in Col. 2, while the intensity of the ghosting line (in counts), is shown in Col. 4. Note that the correction for ghosting is important for many lines, and that some lines have been corrected twice, for both the red- and blue-shifted ghosts.

\begin{tabular}{llllr}
\hline $\begin{array}{l}\lambda_{\text {ob }}(\AA) \\
\text { (ghost) }\end{array}$ & $\begin{array}{l}\text { ratio } \\
\text { (counts) }\end{array}$ & $\begin{array}{l}\text { Ion } \\
\text { (parent) }\end{array}$ & $\begin{array}{l}\lambda_{\text {ob }}(\AA) \\
\text { (parent) }\end{array}$ & $\begin{array}{r}I \text { (counts) } \\
\text { (parent) }\end{array}$ \\
\hline 157.2 & 0.24 & Fe X & 177.24 & 1026 \\
160.6 & 0.17 & Fe XI & 180.49 & 1217 \\
197.9 & 0.04 & Fe XI & 180.49 & 1437 \\
165.3 & 0.15 & Fe X & 184.58 & 530 \\
294.0 & 0.25 & Si VIII & 314.33 & 162 \\
294.8 & 0.25 & Mg VIII & 315.10 & 376 \\
332.5 & 0.03 & Mg VIII & 315.10 & 470 \\
296.9 & 0.68 & Mg VIII & 317.06 & 121 \\
300.1 & 0.05 & Si VIII & 319.78 & 557 \\
336.2 & 0.05 & Si VIII & 319.78 & 585 \\
318.6 & 1.47 & Mg VIII (bl) & 335.30 & 75 \\
744.1 & 0.42 & Ne VIII & 770.41 & 268 \\
\hline
\end{tabular}

be taken into account when analysing GIS spectra are fixed patterning and the problem of ghosts (see Del Zanna 1999 for details). Ghosting was carefully examined before any GIS line was used for calibration purposes. Some "pure" ghosts have been unambiguously identified, and some of the GIS lines could therefore be reconstructed. This has been done by transfering the counts of each ghost to each parent line. However, in many cases the parent lines are contaminated by ghosts of other lines, and therefore their restored intensity should be treated with caution. Table 2 shows an example of the ghosting reconstruction applied to a few GIS lines. Note: a) the line at $744.1 \AA$, the ghost of the Ne VIII $770.41 \AA$, with an intensity more than $40 \%$ of the observed line; b) the line at $318.6 \AA$, a ghost of the $335.3 \AA$ line, is even brighter than the observed parent line; c) the fact that some lines have been corrected for both ghosts.

For the post-recovery observations analysed here, the pointing offset between NIS and GIS has been checked and taken into account. The NIS line intensities have been calculated with multiple broadened line profiles (see CDS Software Note \#53 by W. T. Thompson for details).

\subsection{Intensity calibration factors}

There are several factors which determine the final conversion of the data from photon-events to absolute units. The most important one is the absolute sensitivity eff $(\lambda)$ [counts/photons] as a function of wavelength. The absolute sensitivity of the NI and GI spectrographs is a combination of the reflectivity of the primary mirror, the secondary mirror and the scan mirror, the efficiencies of the gratings, and the quantum sensitivity of the detectors. The wavelength dependence of absolute sensitivity is therefore a combination of the wavelength dependence of all these various factors. The sensitivities were measured on the ground at only a few wavelengths.

The other factors include:

a) the aperture at the telescope $A_{\mathrm{t}}(k)\left[\mathrm{cm}^{2}\right]$, the only spatially-dependent factor in the intensity calibration for NIS. $A_{\mathrm{t}}(k)$ for NIS is a function of the scan mirror position $(k)$ with variations (around a median value of 27 for NIS 1 or 28 for NIS 2) that are only about $2 \%$ for the ARC_CALN study, given the small region rastered. For GIS, the spatial dependency is negligible for the studies analysed, and has been therefore assumed constant with a value of $25.5 \mathrm{~cm}^{2}$;

b) the geometric area $A_{\mathrm{s}}$ available at the slit, which was accurately measured on the ground;

c) the exposure time, which only needs a small dead-time correction.

The values of the $A_{\mathrm{t}}(k), A_{\mathrm{s}}$ factors were assumed to be correct and the same for all the observations (a reasonable assumption). The aim of this study was therefore to deduce from the in-flight observations the first and second order sensitivities eff $(\lambda)$ [counts/photons] as relative values compared to only one absolute reference point, chosen equal to eff $=4.75 \times 10^{-4}$ [counts/photon] at $584 \AA$, in accordance with the Lang et al. (2000) and Brekke et al. (2000) values.

All the CDS spectra presented here are in photon-event units; the spectral line intensities in the figures are in phot $\mathrm{s}^{-1} \mathrm{~cm}^{-2} \operatorname{arcsec}{ }^{-2}$; the intensities in the tables are in ergs $\mathrm{s}^{-1} \mathrm{~cm}^{-2} \mathrm{sr}^{-1}$; the intensity ratio values, both in tables and in figures, are obtained from the intensities in $\operatorname{ergs\mathrm {s}^{-1}} \mathrm{cm}^{-2} \mathrm{sr}^{-1}$.

\section{First step: The effects of the solar variability}

Here, we focus on the NIS-GIS sequences performed near the solar limb. The total of $40(8 \times 5)$ NIS exposures and $180(36 \times 5)$ GIS spectra, for each set of 5 successive NIS and GIS rasters were analysed. An analysis of these successive observations allowed the selection of GIS and NIS averaged spectra emitted by the same spatial regions at times when temporal variations were at a minimum. In order to increase the signal, and reduce the effect of the cosmic rays, averaged GIS and NIS spectra for each mirror position (i.e. for each Solar $X$ ) and for each observation were created from the spatially overlapping region by integrating along Solar $Y$. Line intensities were obtained by using multiple line-fitting IDL routines (see Haugan 1997) on the spectra, removing the "background" intensity. Errors on the line intensities were estimated from the errors of the line fitting parameters. In most cases, these errors are very similar to the Poisson noise (see Del Zanna 1999 for details). Figures such as Fig. 1 have been inspected in order to judge if there were observations with 

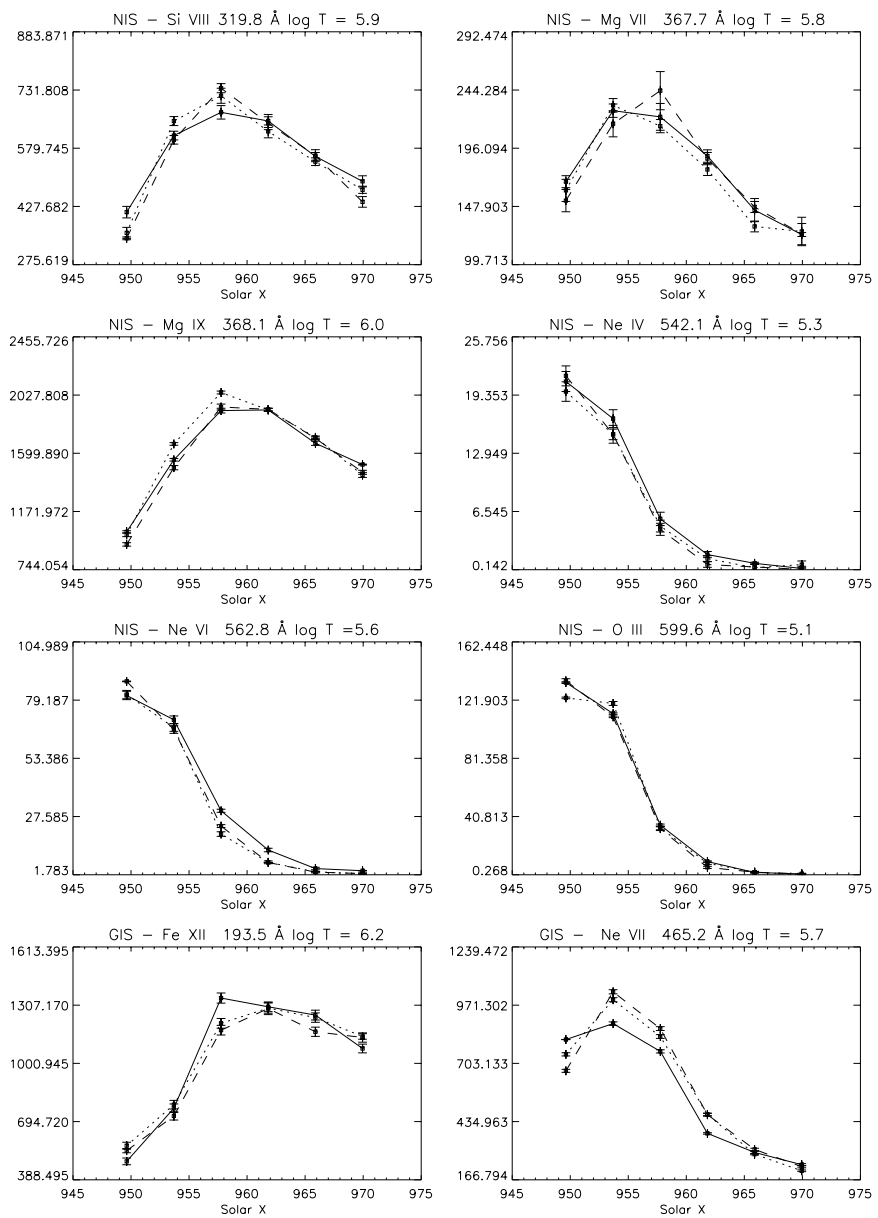

Fig. 1. Intensities of a sample of NIS and GIS lines, as a function of position across the west limb (Solar $X=955$.) - May 4th, 1997. Three successive observations are superimposed, showing the small solar variability with time. Note the limb-brightening in the coronal lines, and the rapid disappearance just off-limb of the "cool" transition region lines such as O III.

brightenings. Where significant variations were found, the corresponding files were excluded from the averaging.

Once this was established for all the observations, an analysis of the time-averaged spectra was performed, with accurate line fitting and background subtraction, for a few spatial positions, typically one on the disc, and one off the limb. The diagnostic method was then applied to each of these spectra, and the information from the data sets combined, to find the relative calibration for each NIS and GIS channel.

\section{Second step: Direct calibration of the overlapping spectral regions and its time stability}

The CDS detectors contain some spectral regions where the same emission lines are observed in two channels, either in first or second order. In these regions, a direct relative calibration, independent of any theoretical assumptions about line intensities, can be obtained.
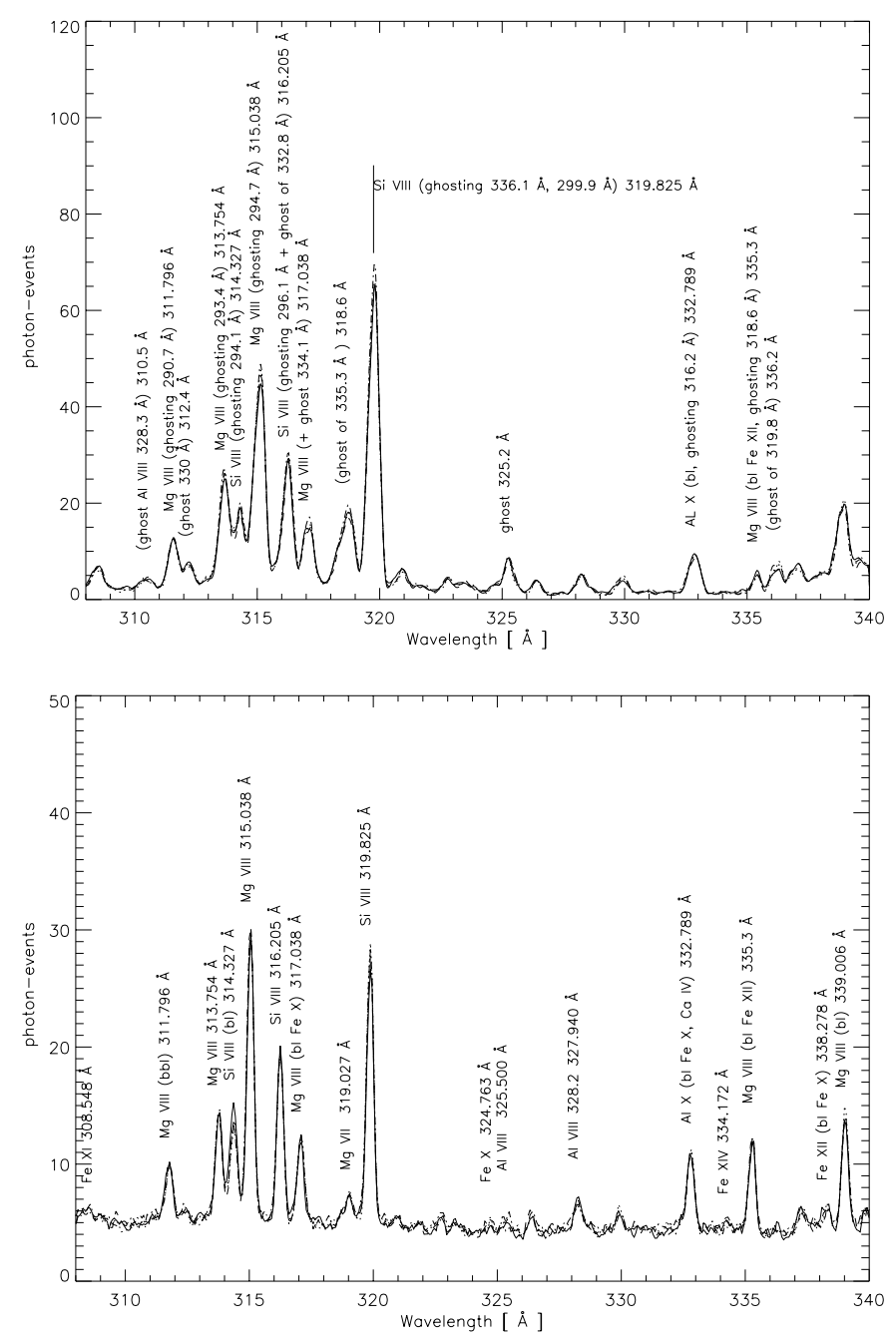

Fig. 2. The on-disc GIS 2 (top) and NIS 1 (bottom) overlapping region, observed on 1997 October 16th, on the disc. Three successive GIS and NIS spectra (taken at about 30 min intervals, at the same spatial location), are superimposed in the figures. Note in each figure the small variations in time, not only in the line intensities, but also in the background, that is higher in NIS 1. This confirms that any differences between the GIS and NIS spectra are not due to temporal variations. When comparing the GIS and the NIS spectra, the most evident differences are due to ghosting in the GIS. Note, for example, the ghost at 318.6 $\AA$, and the ghosting line Mg VIII 335.3 $\AA$.

\subsection{NIS 1 vs. GIS 2}

The best case is given by the NIS 1 and GIS 2 channels, where the $308-340 \AA$ spectral region is observed in first order, as shown in Fig. 2. This figure present time series of spectra superimposed, on the same scale, and visually demonstrate that temporal variations in the line intensities were small. The variations in the "background" scattered light in the NIS 1 channel shown in Fig. 2 are more pronounced.

This direct NIS-GIS comparison undoubtedly shows the presence of ghosts in the GIS spectra. Some of the GIS lines such as the Fe XVI blend at $335.4 \AA$ re almost completely ghosting, and "disappear". Before attempting 

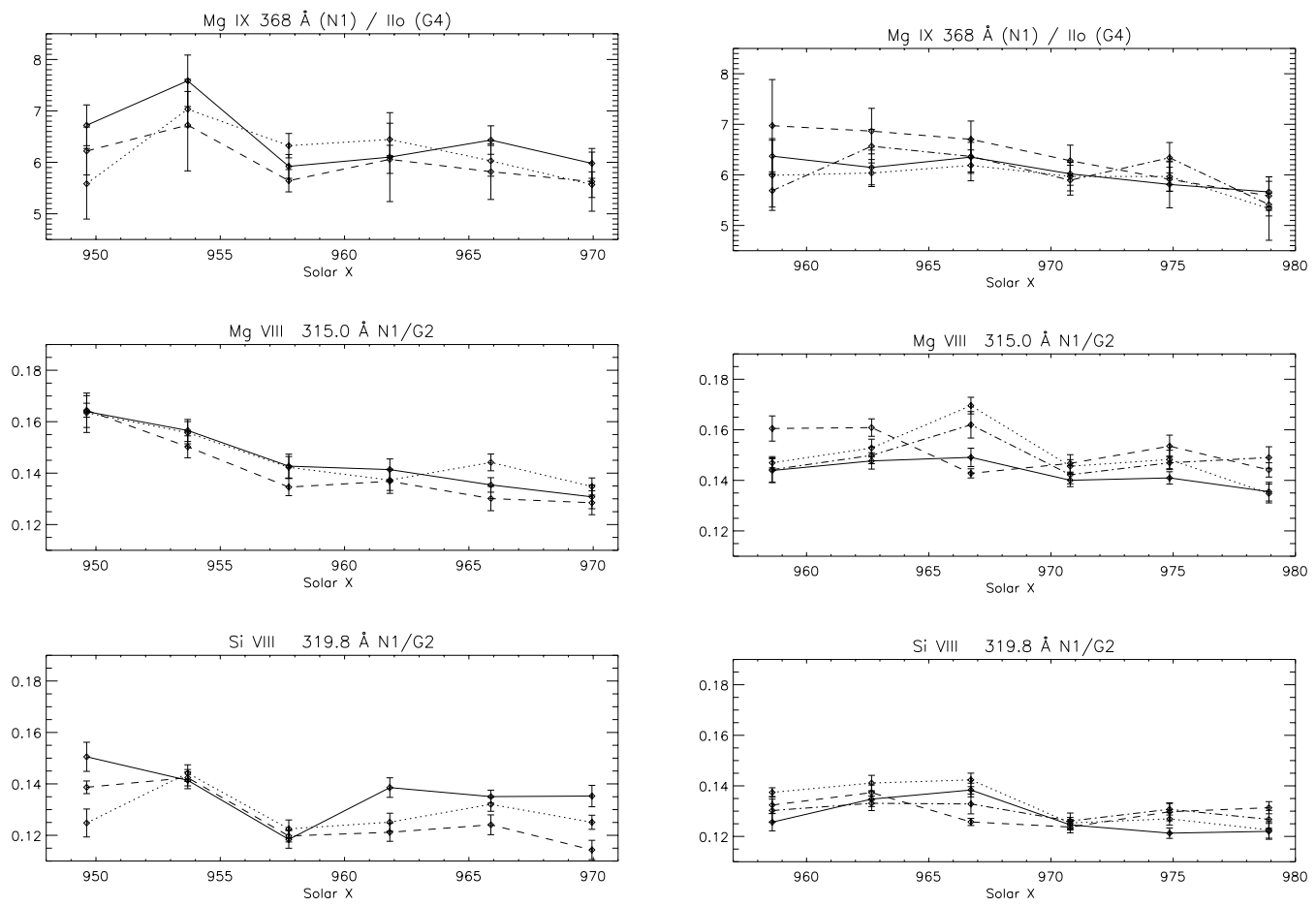

Fig. 3. The ratios of the sensitivities eff $(\lambda)$ (NIS 1 vs. GIS 2 and NIS 1 vs. GIS 4 second order) as derived from the line intensities measured in the NIS and GIS spectra - May 4th (left) and October 16th (right), 1997.

any relative calibration, all the GIS lines that were ghosting had to be reconstructed. Unfortunately, GIS 2 is the channel with the strongest ghosting problems, making the relative calibration difficult. Only a few lines could be reconstructed and used for the direct cross-calibration between NIS 1 and GIS 2. The principal ones are the Mg VIII 315.0 A and Si VIII 319.8 ^. The latter has been chosen as the primary line to scale the relative sensitivities between NIS 1 and GIS 2, because it is the brightest line, is unblended, and only weakly density-dependent. It is also the only one in the overlapping region that creates two weak ghosts in regions free of other lines, and therefore can be accurately reconstructed.

The values of the relative NIS $1 /$ GIS 2 sensitivity in the overlapping region are given in Fig. 3, which shows the ratio of the $\mathrm{Mg}$ VIII and $\mathrm{Si}$ VIII line intensities as a function of time and position. These ratios have been calculated leaving out only the sensitivity $\operatorname{eff}(\lambda)$ in the intensity calibration, NIS and GIS calibration), and are therefore a direct measure of the relative sensitivity between NIS 1 and GIS 2. The NIS channel around $319.8 \AA$ has a sensitivity about a factor 0.12 lower than GIS 2, a value deduced from the off-limb observations. In the ondisc observations, the NIS 1 line intensities appear to be consistently shifted by about $10 \%$, a fact that could be related to the presence of scattered light in NIS 1 . In fact, different selections of the background can easily account for changes in the line intensities up to a factor of $15 \%$. Note that the ratio of the NIS 1 /GIS 2 sensitivities at $315.0 \AA$ is 0.14 , slightly higher. This implies a slightly different wavelength dependence for the NIS 1 and GIS 2 sensitivities in that wavelength region. Finally, note that the relative sensitivities between the NIS 1 and GIS 2 channels did not change significantly between May and October 1997.

\subsection{Other direct calibrations}

No other directly overlapping regions are present in the CDS spectra. However, there are several regions where lines seen in first order in one channel are also seen in second order in another one, allowing again a direct relative calibration, independent of any theoretical assumptions.

\subsubsection{NIS 1 and GIS 2 vs. GIS 4 (second order)}

The most important case is the overlapping region between part of NIS 1 (330-379 $\AA$ ) and GIS 4. Most of the lines seen in the NIS 1 channel (and GIS 2) in first order are observed by the GIS 4 detector in second order. In on-disc observations, the second order lines are heavily blended with first order lines. In off-limb observations, most of the first order lines disappear from GIS 4, and it is possible to directly calibrate a large part of GIS 4 with NIS 1. The main and brightest line used for this purpose is Mg IX at $368 \AA$. Figure 3 shows how constant the relative sensitivity between NIS 1 and GIS 4 was over time. Note that the second order GIS 4 sensitivities at wavelengths $>740 \AA$ are not well constrained and should be taken with caution. 


\subsubsection{GIS 1 vs. GIS 3 (second order)}

GIS 3 also has a relatively high second order sensitivity that creates strong blending between first and second order lines, complicating the interpretation of the spectra. Part of the second order GIS 3 sensitivity was derived from a direct cross-calibration of the same lines seen in first order in GIS 1 (195-221 $\AA$ ), using off-limb quiet sun spectra and active region spectra. Other observations could not be used because the second order lines were either: (1) blended in the GIS 3 spectra with cool lines; (2) not visible, since they are all high-temperature lines of Fe XII, Fe XIII, Fe XIV). Note that the rest of the GIS 3 in second order could only be inferred from theoretical predictions of the lines intensities, with the use of $D E M$ analyses of active regions and off-limb quiet sun regions.

\subsubsection{NIS 1 and GIS 2 vs. NIS 2 (second order)}

Another important issue in the calibration was the assessment of the second order sensitivity in the NIS 2 channel. The quiet sun off-limb observations were used to directly cross-calibrate the NIS 2 second order sensitivity (at the longer wavelengths), with the same lines, observed in first order in NIS 1 and GIS 2 in the 308-316 $\AA$ range (see, e.g. Fig. B.3). The NIS 2 second order sensitivity at shorter wavelengths was directly cross-calibrated using the same lines seen in first order in GIS $2(256-316 \AA)$ and using off-limb active region spectra.

It should be noted that most of these NIS 2 second order lines were regarded as unidentified lines by Brooks et al. (1999). Brooks et al. have presented a comprehensive list of NIS line identifications. Their identifications were based on the analysis of quiet sun spectra, and mostly on line position arguments. Here, the DEM analysis of each spectrum has enabled an accurate estimate of possible blending in each of these lines. This fact, together with the advantage of having observed the same lines in first order in GIS, gives confidence in these identifications, not reported by other authors.

All the line ratios examined have the same averaged values for the dates examined (May, June, and October 1997), thus suggesting that the relative calibration between all NIS and GIS detectors did not appreciably change over six months, in 1997. This gives confidence in the stability of the CDS detectors.

\section{Third step: The assessment of the useful calibration ratios and observations}

The third step in the calibration process was the analysis of the spatial variations of the principal lines observed in all the CDS spectra. The aim was to find out which set of calibration ratios and observations were most appropriate for each ion. In summary, the steps followed were:

- Firstly, variablity of line ratios with spatial location was used to assess blending. Figure 4 shows three examples of line ratios affected by blending in on-disc
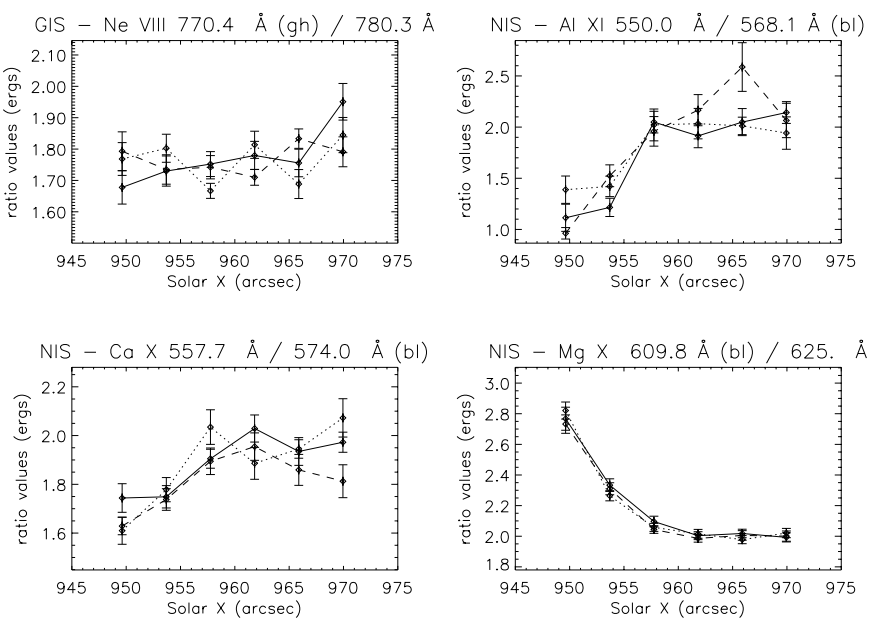

Fig. 4. The intensity ratios of four doublets (Li- and Na-like) seen in NIS and GIS - May 4th 1997. Note that, except for the Ne VIII ratio, one of the lines is strongly blended with a cool line, and it is only off-limb (above Solar $X=960$ ) that the blend disappears, and the calibrated ratios tend toward the theoretical value of 2 .

observations, and for which only off-limb observations are appropriate for calibration purposes. For all the calibration ratios, it was possible to find some observations where blending was reduced to a few percent level. Of course, blending could have been taken into account by using the atomic data, but this introduces a further uncertainty and this method, usually adopted in previous publications, has been rejected here;

- Secondly, it was checked that different observations produced the same ratio values, within the errors. Once this was established, the relative sensitivity between the two wavelengths of the lines that form the ratio can be directly derived once the theoretical intensities of the lines are known;

- Although many of the calibration lines are strictly temperature- and density-insensitive, some have a weak dependence, and it is therefore important to calculate their theoretical intensities at the correct temperatures and densities. Various tests have been performed in order to make sure that the calibration used has a negligible influence on the density and temperature estimate (see Del Zanna 1999 for details). Fortunately, CDS has many diagnostic lines that are close in wavelength and that have been used to derive density and temperature values. Also note that densities and temperatures deduced from most of the ions and line ratios have similar values, giving confidence in the result. Moreover, observations where the plasma is nearly isodensity and isothermal were selected. To reduce temperature effects, theoretical intensity ratios were calculated by using the proper $D E M(T)$ of the source. In this way, calibration ratios that are not strictly temperature- and density-insensitive could be used with confidence; 
- Finally, observations taken at different times show if the relative sensitivity between the two wavelengths was constant or not, independently of its actual value.

A limited number of examples on the above points are now given. The line ratios presented here as samples are grouped in three classes: doublet, coronal, and transition region (cool) lines, and are shown in Figs. 4, 5, and 6.

Among the brightest lines in the CDS spectra are the doublets, which are useful for calibration purposes because their ratio is not density-dependent and the agreement between observations and theory has already been tested in many different solar and stellar studies. Among these, the Fe XVI doublet, seen in the NIS 1 spectra at $335.4 \AA$ and $360.8 \AA$, is particularly important for calibration purposes. Unfortunately, in most cases the doublets observed by CDS are blended with other lines. For example, the observed line at $335.4 \AA$ is a complex blend of Fe XVI, Mg VIII and Fe XII (as discussed below). In normal quiet sun conditions the Fe XVI lines are very weak and the $335.4 \AA$ line is mainly $\mathrm{Mg}$ VIII (plus Fe XII), and not Fe XVI. Only in active region spectra can Fe XVI become the dominant contributor to the observed line. The Al XI, $\mathrm{Ca} \mathrm{X}$ and $\mathrm{Mg} \mathrm{X}$ doublets are also blended, but this time with cool lines, as shown in Fig. 4. The doublets are free of blending only in off-limb spectra, where they become the strongest lines seen across the NIS 2 channel. Therefore, only off-limb observations of these lines can reliably be used for the calibration.

The ratio of intensity of the doublet lines, in the optically thin collisional case, should be 2 . However, this is not necessarily always the case in solar spectra (see e.g. Doschek et al. 1981). For example, Doschek et al. (1998b) found a spatial dependence of the Ne VIII doublet ratio across the limb, with values $\simeq 1.4$ at the limb in coronal holes. This was interpreted in terms of opacity effects. The Ne VIII doublet ratio did not show appreciable spatial variation in the CDS observations analysed, as the example in Fig. 4 shows. Therefore, if departures from the optically thin limit are present, they are very small. Furthermore, the Ne VIII doublet ratio has values close to 2 (the $770.41 \AA$ line has been de-ghosted) thus confirming the optically thin collisional case. It can safely be assumed that no opacity effects are present in the other doublets either, and indeed the CDS intensity calibration has been adjusted in order for these ratios to be 2 .

Beside the doublets, CDS also observes a considerable number of calibration ratios involving coronal lines. In some cases there are some blends with cool lines, and indeed the off-limb observations show a smaller scatter of ratio values. Figure 5 shows some examples. Observations taken at different times show very similar values, thus confirming the stability of the detectors at those wavelengths.

Transition region (cool) lines are "crucial" for a relative calibration of the NIS 1 and NIS 2 channels. Most of the NIS 1 lines are coronal lines, but most of those observed by NIS 2 are formed in the transition region. So these two channels can only be cross-calibrated with the use of the GIS spectra, which observe both transition region and coronal lines in each channel, in particular in GIS 3. Unfortunately, the cool lines exhibit large intensity variations with time (on time-scales of minutes), and only a careful temporal analysis and averaging over a large number of spectra allows these lines to be safely used for calibration purposes.

In conclusion, a sufficient number of good observations and calibration line ratios have been selected, based on the methods explained and as the examples have shown. The observations show that the relative sensitivity of all the CDS channels was very stable in time. The actual values of the relative sensitivities are independent (i.e. largely within the errors of line ratio measurements) of the target source observed and density and temperature estimates. Therefore, the last step in the calibration could be applied.

\section{Fourth step: Application of the spectroscopic method}

The information from all the selected observations was used to constrain the CDS sensitivities, that is to find the wavelength dependence with the use of the diagnostic method. The whole process is an iterative one, summarised below. First, the internal calibration within each NIS and GIS channel was studied, with the use of the diagnostic method. However, only in the NIS 1, NIS 2, GIS 1 and GIS 2 spectra are there pairs of lines emitted by the same ion, which can be used to derive the internal calibration in some spectral ranges. In particular, the number of "calibration" lines in the GIS spectra is so limited that a complete calibration of all the GIS spectra relies on the cross-calibration with the NIS detectors.

The procedure was to obtain first the internal NIS 1 and NIS 2 sensitivity curves, as described in Sects. 9.1 and 9.2. These internal calibrations were derived independently. The procedure was then to cross-calibrate all the other channels, including the second orders, as described in Sect. 9.3, where the final results are presented and compared with the previous calibration studies.

\subsection{The NIS 1 internal calibration}

The off-limb quiet-sun and active region observations have been used to constrain the internal NIS 1 calibration. The NIS 1 internal calibration is presented in Fig. 7. For each pair of lines of the same ion, the ratio of sensitivities was directly derived from the observed intensities and the theoretical ratio value. For each ratio, once one absolute sensitivity value was fixed, then the other is directly obtained. Figure 7 shows the absolute sensitivities derived from various line ratios, by fixing the sensitivity of one reference line for each ion. The sensitivities of the reference lines were chosen in order to fit the continuous curve shown in Fig. 7. A smooth variation of the sensitivities across the channel was sought. For each ion, different reference lines or different absolute sensitivities for the reference line could be used. However, this would simply shift all the points of a 

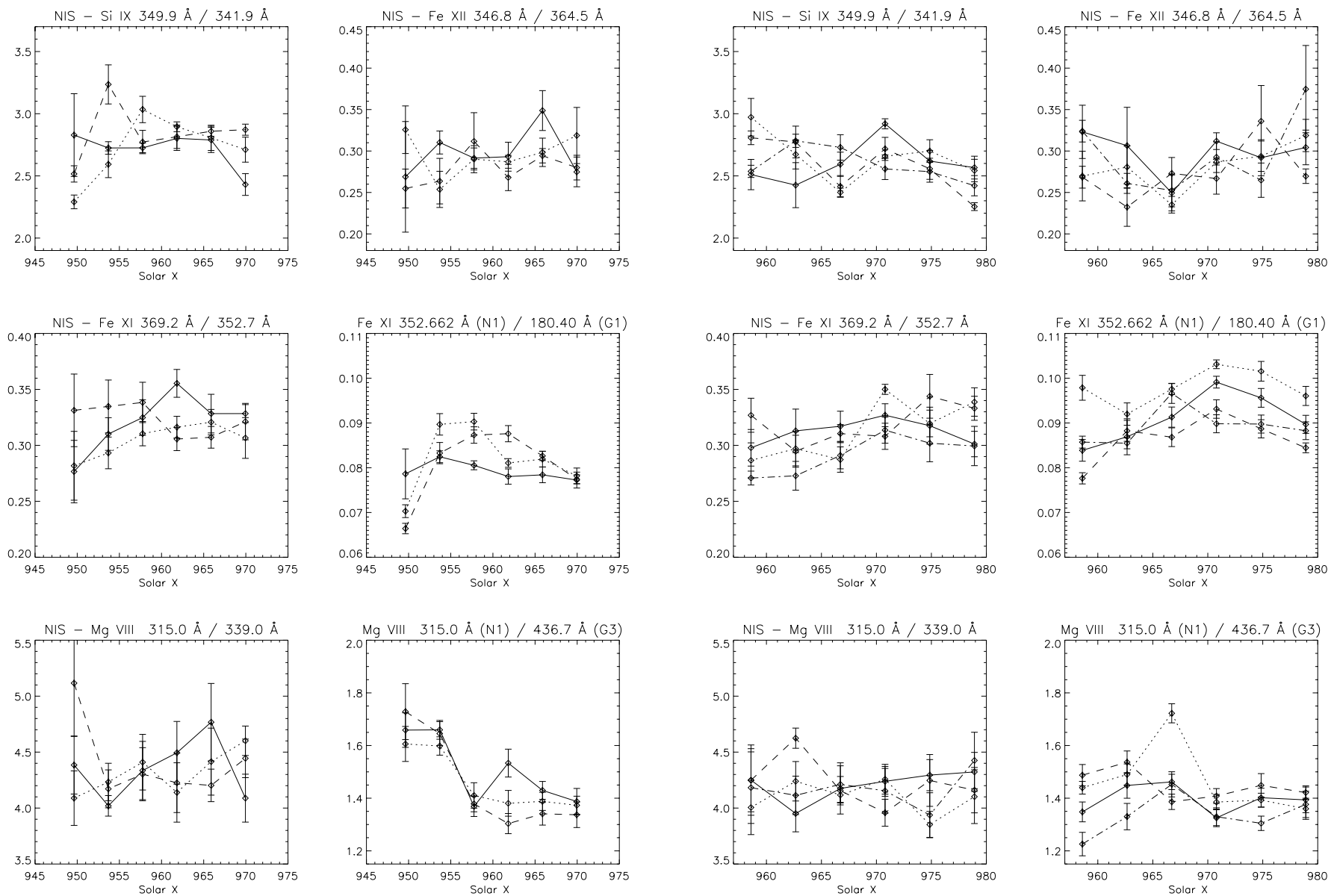

Fig. 5. Some intensity ratios of NIS and GIS coronal lines, used for the NIS/GIS cross-calibration, and for internal calibration within each detector - May 4th (left two columns) and October 16th (right two columns), 1997. The same intensity calibration has been applied for the two dates. Note: (1) that the density is fairly constant, as indicated by the Si IX and Mg VIII ratios (that are density-sensitive); (2) that the relative sensitivities between the presented lines, originating from the same ion, and seen either within the same channel or in different ones, is relatively constant in time and in space, and are therefore usable for calibration purposes; (3) that these ratios have similar values in May and October 1997, giving confidence in the stability of the detectors.

given ion in the figure (since the relative values are fixed), producing disagreement with the points derived from the other ions. The errors on the ratios are calculated from the errors on the line intensities, adding a $10 \%$ error due to independent factors such as those due to the theoretical ratios. These errors therefore do not include all possible systematic errors and should be considered as indicative.

The results from different observations are superimposed in Fig. 7, to show the small scatter in the derived sensitivity ratios, well within the errors.

The selected lines have been chosen because they: 1) are well represented in CHIANTI-SERTS comparisons (Young et al. 1998; Del Zanna 1999); 2) are bright and well resolved; 3) are not blended in the selected observations. Only a few blends have been used, but carefully accounted for. A detailed discussion of the lines can be found in the Appendix. The principal ions used are Fe XVI, Fe XII, Fe XI, Mg VIII.

The apparently unconstrained regions of the sensitivity curve are in fact constrained by other lines, which are shown in Fig. 10. Among them are lines from O III,
Fe XIII, Fe XIV, Fe XV, that span almost the whole NIS 1 channel, and that were used in conjunction with lines seen in the other CDS channels, to constrain the NIS sensitivity, in relative terms. For example, the group of O III/N III lines at $374 \AA$ has been used in conjunction with the O III lines seen in GIS 4 and NIS 2 in on-disc spectra. These lines also indicate an increased sensitivity at longer wavelengths. The downturn at the end of the detector should be taken with caution, since it is only constrained by a weak blend of Ca IX and Na VII lines at $378 \AA$. Figure 7 also shows the previous calibrations for comparison:

a) The first CDS standard calibration, which was based on the ground calibration of Bromage et al. (1996) (Lang et al. 2000 have later provided additional points). It is evident that the ground calibration was obtained at only a few wavelengths. The two measurements around $360 \AA$ suggested a constant sensitivity in this region;

b) The Landi et al. (1997) calibration, increased by a factor of 2 (they suggested a factor ranging between 2.5 and 5); 

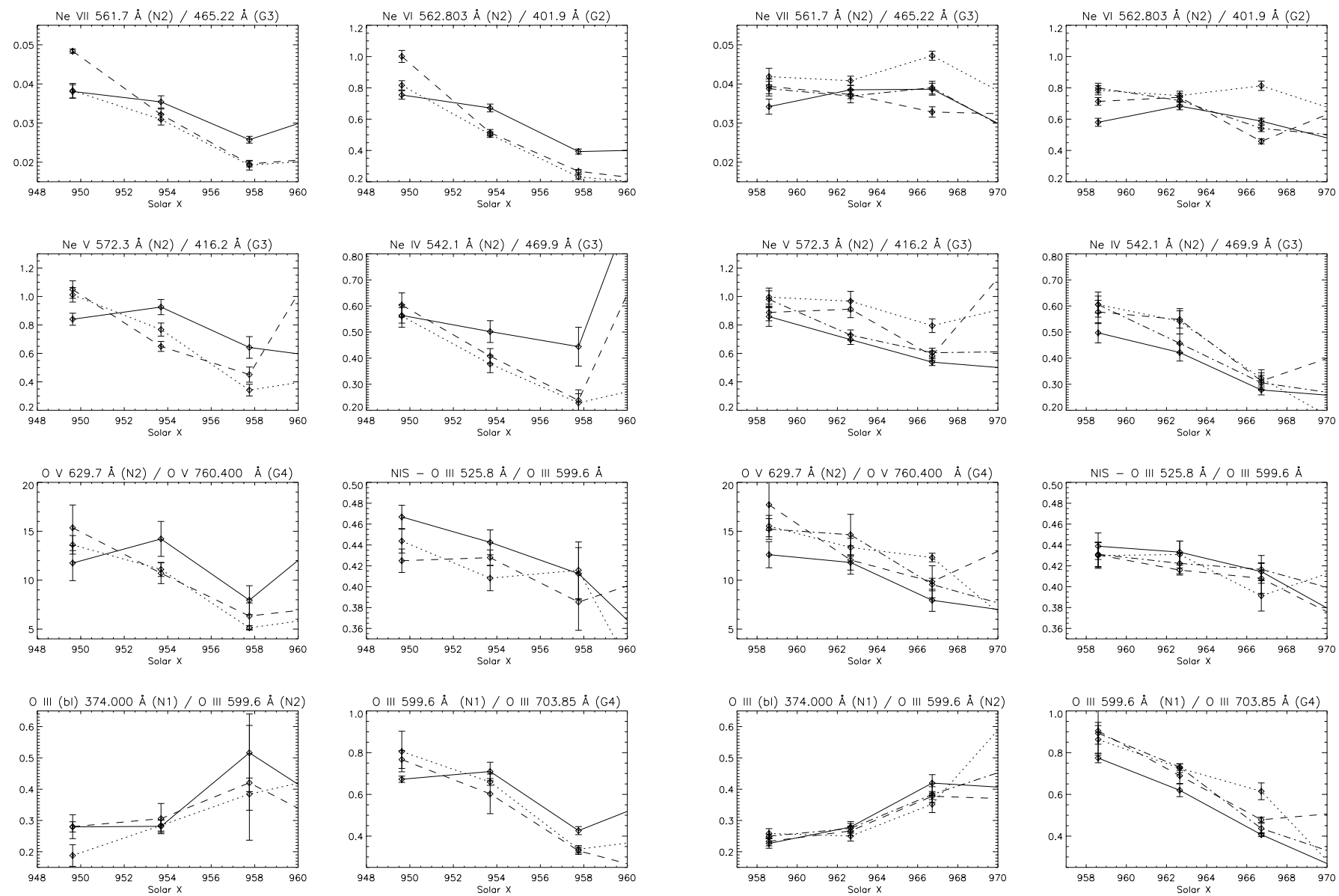

Fig. 6. Some ratios of intensities of NIS and GIS transition region lines, useful for the NIS/GIS cross-calibration, and for internal calibration within each detector - May 4th (left two columns) and October 16th (right), 1997. The same intensity calibration has been applied for the two dates. Note: (1) how temporal variations are small, considering that these cool lines always present large intensity variations; (2) how close the ratio values are for the two different dates, confirming that the relative sensitivities for the lines shown have been constant between May and October 1997. These lines (among others not shown here) are therefore suitable for relative calibration purposes, for these observations. Only the values on the disc were used for the calibration (the first point, at Solar $X \simeq 949^{\prime \prime}, 958^{\prime \prime}$ ), since these lines quickly disappear above the limb (see Fig. 1), and their intensity could be affected by stray light.

c) The second NIS standard calibration. The absolute value at $368 \AA$, where the brightest NIS 1 line is, was derived by Brekke et al. (2000) with a reported accuracy of $25 \%$. However, the coarse resolution $(\simeq 5 \AA)$ of the rocket flight spectrum, and the low signal-to-noise, did not allow a measurement of the wavelength dependence for NIS 1 , and the quoted uncertainty from Brekke et al. (2000) is $45 \%$ in the remainder of the spectrum;

d) The third NIS standard calibration.

In conclusion, excellent agreement is found between the calibration presented here and the other two independent in-flight studies based on rocket flights in 1997 (Brekke et al. 2000; Thomas et al. 1999). In particular, the values of the calibration presented here almost coincide at $368 \AA$ with the only reliable absolute NIS 1 measurement of Brekke et al. (1.86 $\pm 0.46 \times 10^{-4}$ counts/phot). The SERTS-97 was accurately calibrated on the ground against primary standards, and the comparison between the CDS and SERTS-97 line intensities (Thomas et al. 1999) gives a direct absolute measure of the CDS sensitivities, shown in Fig. 8. The SERTS-97 covered the spectral region from 310 to $355 \AA$. Very good agreement with the calibration proposed here is found, in particular at $320 \AA$ and in the region $339-353 \AA$. The main disagreement concerns the lines that have been labelled, Al X $332.8 \AA$, Fe XIV $334.2 \AA$, and Fe XVI $335.4 \AA$. The CDS and SERTS-97 observed a very bright active region, and these lines were the most prominent ones in the SERTS-97 spectrum (indeed the measurement error is very small). The observations were made at a location with steep spatial gradients in the hottest lines, and any small spatial misalignment between the two instruments would mostly affect these three lines, that are the higher temperature lines in the SERTS-97 spectrum (R. J. Thomas 2001, priv. comm. - further work is in progress).

On the other hand, the NIS ground calibration data imply a much lower sensitivity than that one measured in flight, by factors of more than 2 across the channel. These large differences remain unexplained, but could be due to a misalignment between the CDS instrument and the 


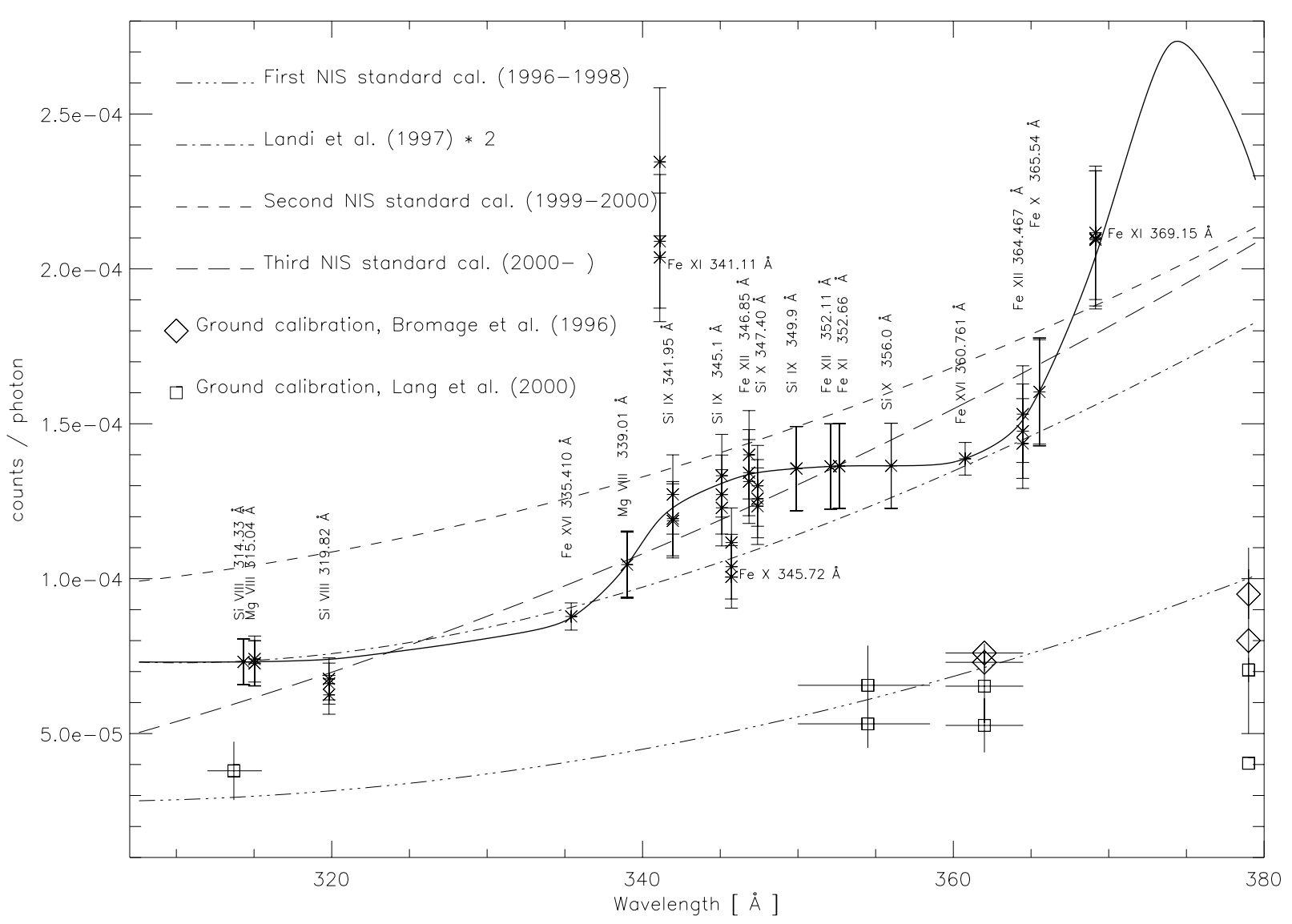

Fig. 7. The proposed CDS NIS 1 sensitivity (solid line), compared with the other calibrations. The asterisks indicate the scaled values of the sensitivities, as derived from ratios of lines of the same ion observed within this detector.

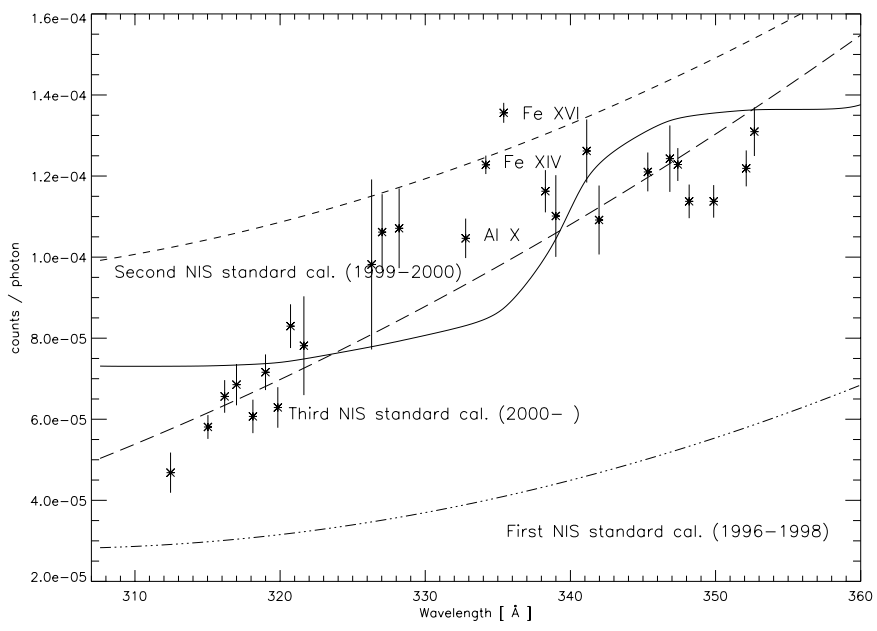

Fig. 8. The proposed NIS 1 sensitivity (solid line), compared with the absolute values derived from the CDS / SERTS97 cross-calibration (stars), and the previous NIS 1 standard calibrations.

calibration source (J. Lang 2000, priv. comm.). Neither the the ground calibration measurements nor the theoretical predictions of the sensitivities are in accordance with the direct in-flight measurements.

\subsection{The NIS 2 internal calibration (first order)}

Unlike the NIS 1 channel, not many different lines emitted by the same ion are seen in NIS 2. Moreover, some of these lines are complex blends between "cool and hot" lines (as with second order lines). The lines useful for calibration can be grouped into two classes: the coronal and the transition region (cool) lines. The on-disc quiet sun NIS observations analysed have been used to constrain the sensitivity using the cool lines, while the off-limb quiet sun observations were used to study the sensitivities using the coronal lines. Active region observations were discarded because of blending with second order lines.

The results are presented in Fig. 9, in the same way as in Fig. 7. A detailed discussion of the lines used can be found in the Appendix. The principal ions used are O III, O IV, He I, Ca X, Mg X, Al XI. As in the NIS 1 case, other lines have been used to obtain a consistent calibration relative to the other CDS channels. These lines are shown in Fig. 10. As an example, the upturn at the longer wavelengths has the effect of reducing the calibrated intensity of one of the brightest lines in NIS 2, O V $629.732 \AA$, by almost a factor of 2 . Only with this upturn is it possible to find a good agreement with the multiplet $\mathrm{O} \mathrm{V}$ lines seen in GIS $4 \simeq 760 \AA$. 


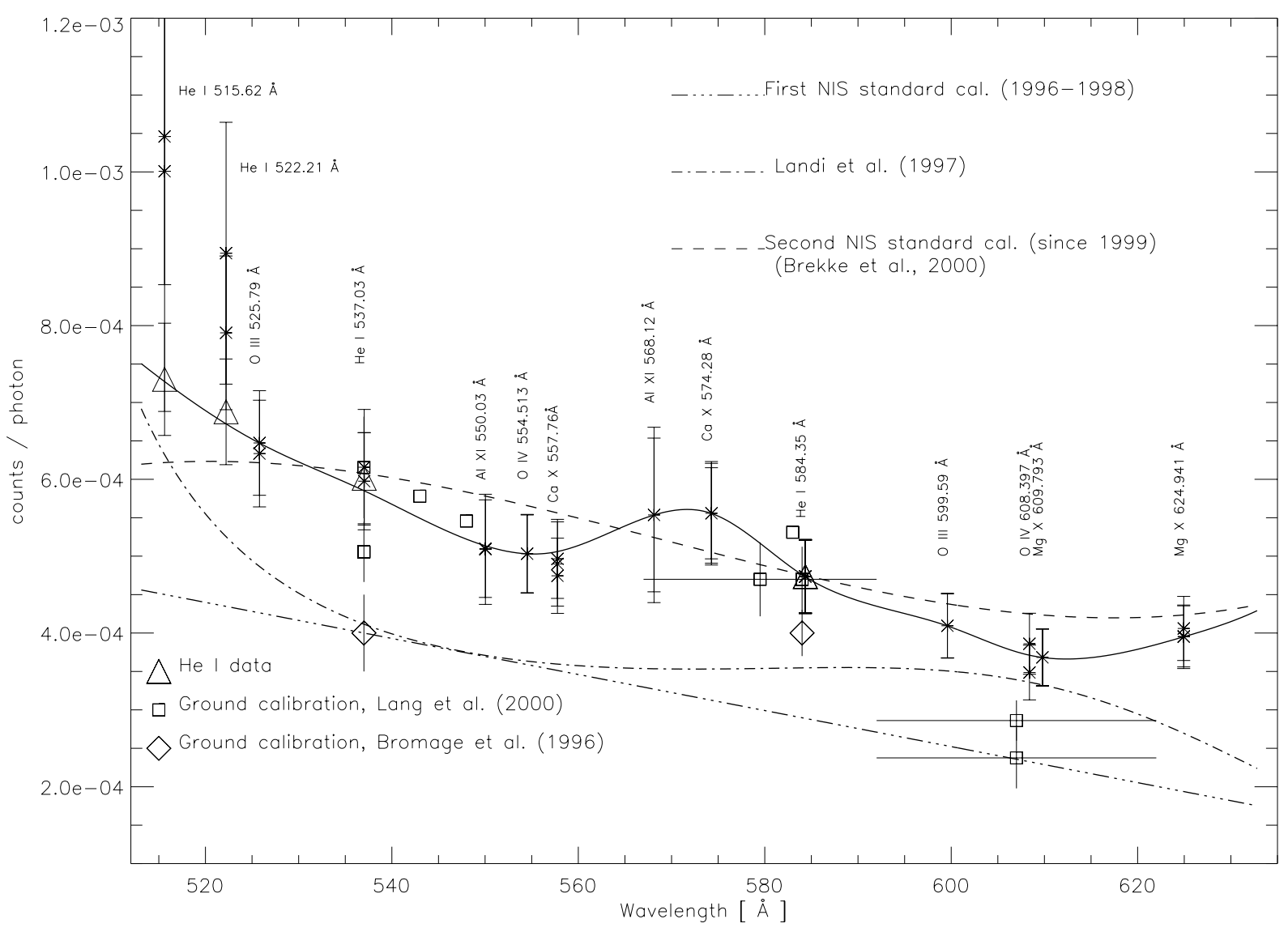

Fig. 9. The proposed CDS NIS 2 sensitivity (solid line), compared with the other calibrations. Notations as in Fig. 7.

As in the NIS 1 case, the result is compared with the other calibrations:

a) The first CDS standard calibration. The most reliable measurements obtained there were from the wide slit He I $(584.3,537.0 \AA)$ observations. A number of different observations resulted in a mean sensitivity value equal to 0.00040, at both wavelengths (Bromage et al. 1996). The measurement at $537 \AA$ was adopted for the first CDS standard calibration, and a linear variation along the detector was introduced to explain the O III $(525.8 / 599.6 \AA)$ ratio observed in-flight;

b) The Landi et al. (1997) calibration. The main features are an almost constant sensitivity along the central part of the channel, with an increase at the shorter wavelengths and a downturn at the longer ones;

c) The second CDS standard calibration. We recall that this calibration is based on the Brekke et al. (2000) study. For the NIS 2 channel, in fact, the signal was sufficient to allow an estimate of the background intensity. The main features are an increased absolute sensitivity, by a factor of 1.6 compared to the earlier ground calibration, a rise at the high wavelength and a downturn at wavelengths below $530 \AA$. The cited accuracy in terms of absolute values ranges from $15 \%$ at $584 \AA$, to $25 \%$ at either end of the spectrum. However, additional uncertainties could be due to the fact that the rocket flight measured the emission from the whole sun, while the CDS observations were not simultaneous and "spatially under-sampled" the sun; d) The revised ground calibration data of Lang et al. (2000) are also displayed in the figure. The main changes, compared to the previous ground calibration, are an increase in the absolute values of the wide slit He I data. In addition other measurements (with wide and narrow slit) were included. The revised absolute value at $584 \AA$ is in excellent agreement with the value derived by Brekke et al. (2000), suggesting that the NIS 2 sensitivity did not appreciably change between the ground calibration and 1997 (unlike NIS 1).

The only direct in-flight absolute calibration study is that of Brekke et al. (2000). In considering a detailed comparison with the diagnostic study presented here, it should be noted that the coarse resolution $(\simeq 5 \AA)$ of the rocket flight spectrum limited the evaluation of the instrumental background, increasing the uncertainty. The spectra have been binned into six wavelength regions, and the background estimation was very difficult. Therefore, although the differences between the in-flight calibration study of Brekke et al. (2000) and the NIS 2 relative calibration presented here appear considerable, in reality the two studies are not in disagreement at all. In fact:

- The downturn at wavelengths below $530 \AA$ is regarded as uncertain by Brekke et al. (2000), because of low signal, and no comparison can therefore be made with the upturn in the calibration presented here; 
- The region 590-610 $\AA$ proved difficult to fit and was not used by Brekke et al. (2000). Again, no comparison can therefore be made with the decreased sensitivity at $610 \AA$, derived here from O III and O IV lines;

- The region between the He I 537 and $584 \AA$ shows more variation in sensitivity in the calibration proposed here. However, one of the six wavelength bins (the central one) in the Brekke et al. (2000) study spanned the 545-575 $\AA$ range. Actually, the averaged sensitivity in the $545-575 \AA$ range is similar in the two studies.

Note that neither the ground calibration measurements nor the theoretical predictions of the sensitivities (see Lang et al. 2000) are in accordance with both the direct in-flight results of Brekke et al. (2000) and those presented here. Let us consider first the predicted sensitivities. They are not in agreement with the in-flight measurements (e.g. the upturn at the longer wavelengths) nor even with the ground calibration measurements. Some of these discrepancies can be explained by assuming that some ground measurements are unreliable. For example, the measurement centred at $610 \AA$ was rejected from the earlier ground calibration because it was considered that it could be underestimated by about 100\% (Bromage et al. 1996). However, other measurements should be reliable, and therefore the discrepancy is not explained. Let us now consider the ground calibration measurements compared with the direct in-flight calibrations. Again, significant discrepancies are found. Measurements such as that one at $610 \AA$ could be discarded as mentioned. However, measurements such as those of the He I lines were regarded as the best ones over the whole of the CDS in the ground calibration, because of many reasons (they were performed with the wide slit, had good signal, etc.).

Finally, it is interesting to note that the calibration proposed here would partly explain the discrepancies that were found when the SUMER detector A and the NIS calibrations were compared in-flight at 584, 610,625 (see Pauluhn et al. 1999).

\subsection{The cross-calibration between all the NIS and GIS channels, first and second order}

Figure 10 shows the CDS first and second order sensitivities that were derived from the cross-calibration ratios and from pre-recovery data. As in the previous cases, all the line intensities within one ion have been rescaled with a single scaling factor, to remove any inter-ion systematic effects. We recall that these effects mainly depend on the emission measure and element abundances adopted, and do not affect the result. A summary of the lines used follows below, while readers interested in the details will find them in the Appendix. Most of the lines are very well reproduced, well within $20 \%$. Also note that some spectral regions are better constrained than others. The regions at the edges of the detectors have been the most difficult to estimate, and in some cases no reliable lines were found,
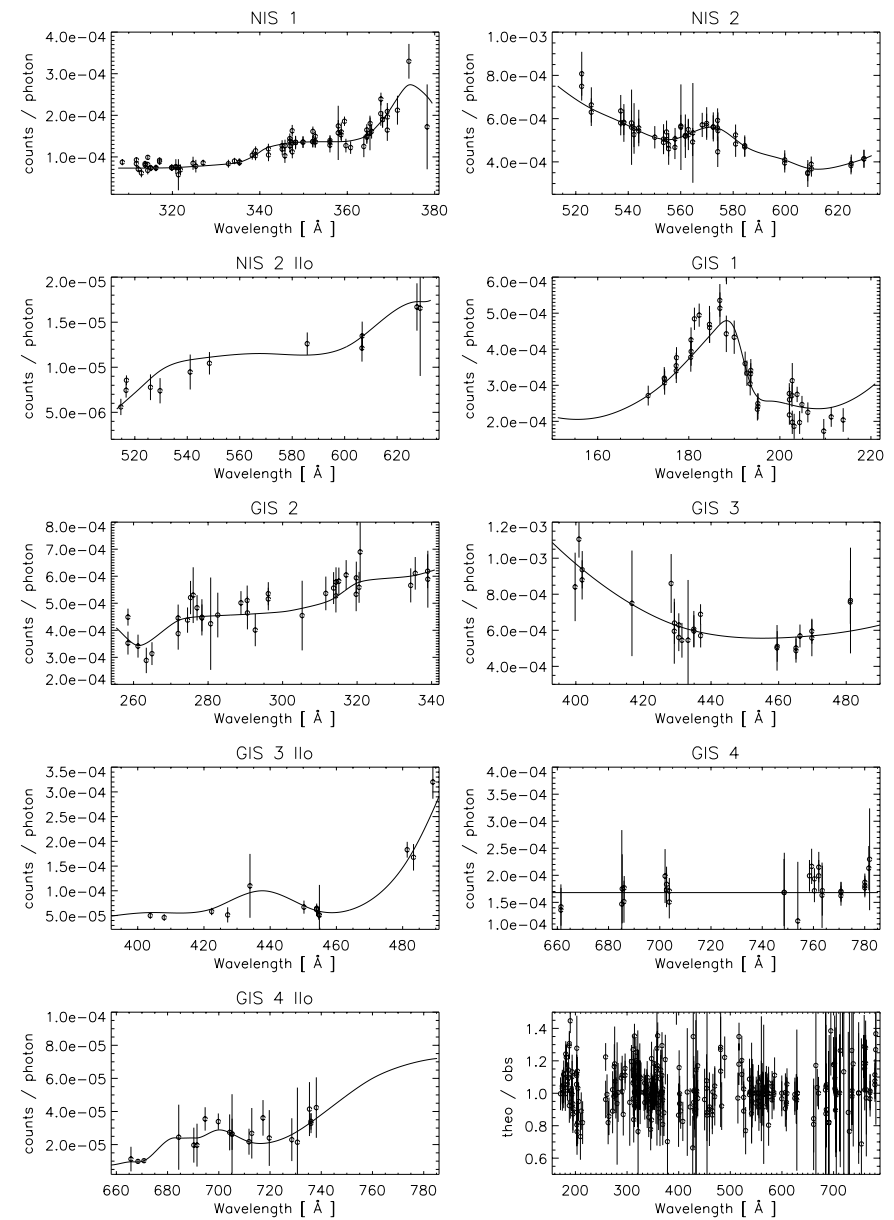

Fig. 10. The CDS NIS and GIS sensitivities (solid lines), first and second order, as derived from pre-recovery observations. The absolute sensitivities derived from all the line ratios used are superimposed. The bottom right plot shows the ratio of the theoretical vs. the observed line intensities for all the line used.

leaving the sensitivities uncertain there. Figure 11 shows the same CDS first and second order sensitivities in a logarithmic scale, to compare them with previous studies.

\subsubsection{NIS 1 vs. NIS 2}

For the NIS 1 and NIS 2 cross-calibration, the diagnostic method has large uncertainties, since in NIS 1 and NIS 2 there are only a few lines emitted by the same ions. Lines from Ne IV, Ne V, O III, N III produce results consistent with those obtained from the other cross-calibrations.

\subsubsection{NIS 1 vs. GIS 2}

The cross-calibration in the overlapping region is straightforward (except for the ghosting problem, as already described), since both channels detect the same lines. Other lines in the GIS 2 channel were used to derive the wavelength dependence. These include lines from $\mathrm{Fe} X I V$, Fe XV, Fe XVI, Si X, Si VIII, Mg VII, using off-limb NIS/GIS and GIS active region observations. 


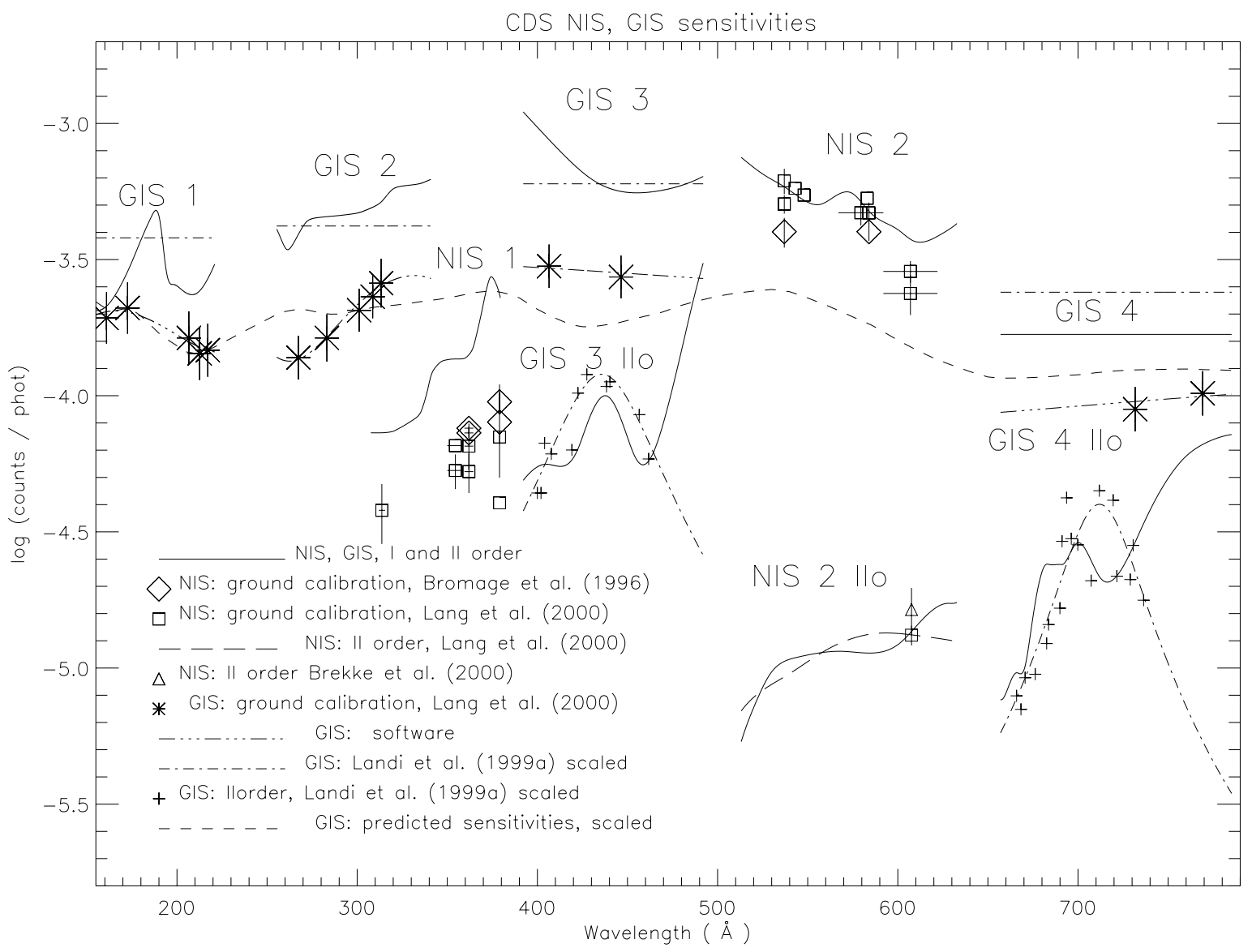

Fig. 11. The CDS NIS and GIS first and second order sensitivities proposed here (solid lines). All the pre-launch NIS and GIS measurements are also displayed. The overall GIS predicted efficiency, as a function of wavelength, is plotted with a dashed line (arbitrary scale - see text). For the NIS 2 second order, the value found by Brekke et al. (2000) is also shown. For the GIS, the sensitivities derived by Landi et al. (1999a) for the first and second orders are also displayed (absolute values scaled by a factor of 2). For the GIS 3 and 4 second orders, the points used by Landi et al. (1999a) to derive the curves are also displayed (crosses), to show that those data are broadly consistent with the curves presented here.

\subsubsection{NIS 1 vs. GIS 1}

This cross-calibration is relatively straightforward, since NIS 1 and GIS 1 observe several Fe X, Fe XI, and Fe XII bright lines, in both the on-disc and off-limb case. In active region spectra, there are also bright Fe XIII and Fe XIV lines. Density-sensitivity is an issue for many of these lines, so the off-limb quiet sun spectra were used, because the plasma has an almost uniform density there.

\subsubsection{NIS 1 vs. GIS 3}

The calibration of the central part of GIS 3 was carried out using the bright Mg VIII and Mg VII lines visible in the 429-437 $\AA$ region with the Mg VIII and Mg VII lines seen in NIS 1 . The quiet sun off-limb observations are the most suitable, because the ghosting of the GIS lines can be checked (see below), and because blending with second order lines is negligible. The Mg VIII lines are more suitable than the Mg VII lines, because they vary much less with time.
GIS 3 also observes some Mg IX lines (439.18, 441.20, $443.973,448.29 \AA$ ) that could be used in conjunction with the bright resonance $368.07 \AA$ line observed in NIS 1 . However, although excellent agreement among the GIS lines was found, no agreement with the $368.07 \AA$ line could be found, confirming the SERTS-89 results described by Young et al. (1998).

\subsubsection{NIS 1 vs. GIS 4}

The first order cross-calibration was not really possible, since only a few lines (with temperature and density effects) could be used: Mg IX 706.06, 749.55 $\AA$ (GIS 4) with the $368.07 \AA$ (NIS 1), and the weak Mg VIII $782.34 \AA$, with the Mg VIII lines seen in NIS 1.

\subsubsection{Cross-calibration between NIS 2, GIS 3, GIS 4}

Since most of the NIS 2 lines are transition region lines, and the second order sensitivity is non-negligible, only the quiet sun on-disc observations could be used. Careful examination of any temporal changes had to be considered 
here. The lines used for both the internal GIS 3 and GIS 4 calibration, and for the cross-calibration are mainly from O III, O V, Ne IV, Ne V, Ne VI, Ne VII, S IV.

\subsubsection{Other cross-calibrations}

A few other indirect cross-calibrations were possible, using for example lines that have similar contribution functions, emitted by different elements, provided it was possible to independently check the relative element abundance. Just as a simple example, the $\mathrm{Mg} / \mathrm{Si}$ abundance can easily be checked using the two 315.04 (Mg VIII) and 316.205 (Si VIII) A lines, observed in NIS 1 (in GIS 2 the Si VIII 316.20 has serious problems of ghosting and contaminations and cannot be used). These lines are close in wavelength and therefore their ratio of intensity does not depend on the calibration. Once the $\mathrm{Mg} / \mathrm{Si}$ abundance ratio is known, then the Si VII line seen in GIS 2 at $275.5 \AA$ can be used to cross-calibrate that spectral region with all the other regions (GIS 3, NIS 1) where all the Mg VII lines are seen.

\subsubsection{The post-recovery case}

The results obtained from the May 1999 observation are displayed in Fig. 12. This on-disc observation did not allow an accurate measurement of the second order sensitivities, with the exception of one for GIS 4 (based on the bright Mg IX $368.0 \AA$ line, seen in first order in NIS 1). This measurement (not shown in Fig. 12) shows excellent agreement (within few \%) with the pre-recovery case. Regarding the first orders, it should be mentioned that GIS 2 was switched off. Moreover, the reduced spectral resolution of the NIS 1 spectra (see Fig. B.2) only allowed a limited number of lines to be used. Discrepancies in some GIS lines have been found. However, Fig. 12 shows that the post-recovery data of this observation are consistent with the pre-recovery relative sensitivities.

\subsubsection{Summary of the new NIS and GIS calibration}

The main features of the calibration presented here, compared to the previous studies are listed below. Note that the cross-calibration was relative to an absolute value at $584 \AA$ chosen to be in agreement with the Lang et al. (2000) value.

- Much increased sensitivities in NIS 1 and in all the GIS channels, compared to the pre-launch values. For the NIS 1 case, the factor is about 2 (1.9 at $317 \AA$ and 2.2 at $362 \AA$ ), while for the GIS case the factors are more variable ( 1.45 at $206 \AA$, GIS $1 ; 2.8$ at $283 \AA$, GIS 2; 2.9 at $406 \AA$, GIS $3 ; 1.9$ at $732 \AA$, GIS 4). We have already mentioned the good agreement for NIS 1 with other in-flight studies. For GIS, it is interesting to note that the calibration proposed here would explain the discrepancies that were found when the SUMER and the GIS calibrations were compared in-flight at $770 \AA$,
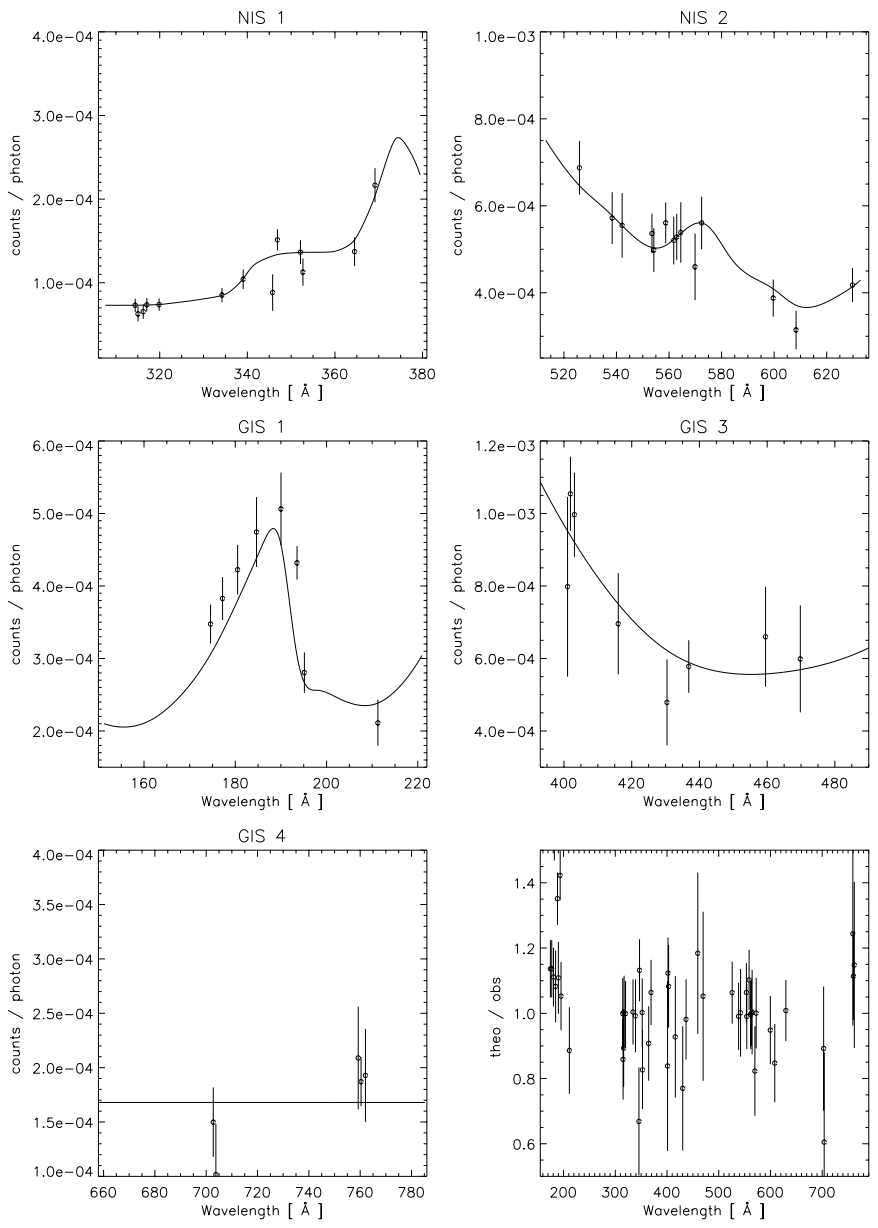

Fig. 12. The CDS NIS and GIS pre-recovery sensitivities as in Fig. 10, with the values derived from the post-recovery observations of May 1999. The bottom right plot shows the ratio of the theoretical vs. the observed line intensities. No significant changes in the calibration are present.

and where a factor of 2 was also found (Pauluhn 2001, priv. comm.);

- Excellent agreement (within few percent) of the second order NIS 2 sensitivity at $608 \AA$ (He II $304 \AA$ line, $1.36 \times 10^{-5}$ ) compared to the only pre-launch measurement by Lang et al. $\left(2000 ; 1.32 \pm 0.11 \times 10^{-5}\right)$. The measurement of Brekke et al. (2000) is about $20 \%$ higher $\left(1.64 \pm 0.12 \times 10^{-5}\right)$, while the SERTS-97 flight has provided a value of $1.68 \pm 0.25 \times 10^{-5}$ (R. Thomas, priv. comm.). Some differences with the predicted second order sensitivities at the other wavelengths are found;

- If average values of the relative sensitivities between all the GIS channels are considered, good agreement is found with the Landi et al. (1999a) values (Fig. 11 shows the sensitivities derived by Landi et al. scaled by a factor of 2 );

- The wavelength variations of the sensitivities, within each GIS channel, can be regarded as consistent with the few pre-launch GIS measurements, except for GIS 2 where a steeper wavelength dependence was measured on the ground. Also, these variations 
follow, in most spectral regions, the predicted sensitivities (shown in Fig. 11, and described in detail in Bromage et al. 1996 and in Lang et al. 2000). These predicted sensitivities were evaluated from the reflectivities of the telescope, the scan mirror, the grating, and the efficiencies of the detectors, and are similar for both GIS and NIS, since the major contribution is given by the telescope and the scan mirror reflectivities, which are common to both spectrometers. Note that the absolute values of the predicted sensitivities are about a factor of 10 higher than the values presented here;

- The shape of the second order curves suggest that most of the sensitivity variations are probably due to the telescope and scan mirror reflectivities, rather than to the grating efficiency in second order. In fact, their wavelength variations in second order (e.g. most of NIS 2 IIo) are similar to the corresponding first order variations (e.g. most of GIS 2).

Densities and temperatures of different observations were calculated with the calibration presented here, and with the second standard NIS calibration (1999) for comparison. When lines close in wavelength are used (e.g. densities derived from Si X 347.4, 356.0 A lines), the differences are small, within the errors. However, in many other cases (e.g., the densities derived from Fe XIV 353.8, $334.2 \AA$ or $\mathrm{Mg}$ VII 319.0, $367.7 \AA$ lines) differences of up to a factor of two were found. If the first NIS standard calibration (1996-1998) is applied, even larger differences can be found.

If lines emitted by different elements are used for calibration purposes, then in some cases large discrepancies (by factors of 2-3) between theory and CDS observations were found. In most of the cases, these disagreements are not due to instrument calibration problems, but can be explained by inaccuracies in the adopted ionization equilibrium calculations (see Del Zanna 1999 for details). This therefore stresses the importance of using a calibration method that does not depend on ionization equilibrium calculations.

\section{Conclusions}

The CDS instrument has for the first time provided the opportunity to observe the solar corona with high spectral and spatial resolution over an extensive wavelength range (150-800 $\AA$ ). This enables a large number of EUV emission lines, from a wide range of ions and elements to be recorded in a single observation. For a proper application of spectroscopic diagnostic techniques, however, an accurate calibration of the instrument is required. Given the characteristics of the current EUV instruments, a calibration performed on the ground should only be used as a preliminary estimate, and must be reviewed with an inflight calibration. Here, the methods and the difficulties for an in-flight calibration of an EUV spectrometer such as CDS have been described in detail. It is advisable that similar in-flight calibrations are also used for future spectroscopic missions, in order to monitor the instruments performance with time. Long term variations of the absolute calibration should still be checked with rocket flights.

For the first time, the in-flight relative sensitivities between all the six CDS detectors (and the second orders for NIS 2, GIS 3 and GIS 4) have been fully determined. This has been achieved thanks to the accuracy and completeness of the CHIANTI atomic database, and by applying a spectroscopic method to a large number of specially designed observations. Excellent agreement was found with the results of the two independent calibrations based on rocket flights flown in 1997 (Brekke et al. 2000; Thomas et al. 1999), thus showing the validity of the method adopted. Our work provides relative calibrations along the detectors to a higher resolution in wavelength.

Most of the lines are well represented to within $\sim 10 \%$, when lines emitted by the same ion are considered. The overall consistency of our results is demonstrated by the comparison between synthetic and observed spectra. Given all the uncertainties in the atomic physics, the observations, and the ghosting problem in the GIS spectra, this is an excellent result.

Despite all the complications of using the Sun as the calibration source that have been described in detail, we have found a considerable number of line ratios that are approximately constant, independent of the solar region observed. These line ratios can therefore be used also in the future for calibration purposes. The example of the He I and He II lines is particularly important, since these lines are the brightest in the EUV wavelength range. The values of the calibration ratios that have been presented here are largely consistent with both theoretical calculations and with previous well-calibrated data.

The calibration method was applied to data taken at different times, in order to monitor the in-flight CDS performance. Temporal variations over a period of six months in 1997 were studied in detail, showing that the relative calibration between the CDS channels did not appreciably change. This confirms the excellent CDS in-flight stability. Analysis of post-recovery data taken in May 1999 has also indicated that no significant changes of the relative calibrations between the CDS channels took place, a quite remarkable result.

Acknowledgements. G. Del Zanna work has been supported by a scholarship from the University of Central Lancashire and by PPARC. The authors thank: the whole of the CDS team (and in particular R. A. Harrison, C. D. Pike and W. T. Thompson) for their support in the planning and operation of the instrument and for useful comments; J. Lang, for providing the data of the pre-launch calibration and for useful comments; S. Parenti for providing an active region spectrum; R. J. Thomas for stimulating discussions and for providing the SERTS-97 data; H. E. Mason for continuous support and suggestions; S. W. McIntosh for his valuable comments about major simplifications to improve the readability of the paper. 


\section{Appendix A: Details on the lines used for the calibration}

\section{A.1. NIS 1}

\section{Fe XVI (335.41, 360. А)}

Intensities from active region observations were used. Only in such observations is the $335.4 \AA$ line entirely due to Fe XVI. Otherwise, this line is strongly blended. A blend with a Mg VIII 335.253 $\AA$ line is commonly reported. However, the Fe XII energy levels reported by Jupen et al. (1993) indicate also the presence of a densitysensitive Fe XII line at $335.339 \AA$. The presence of this line (too close to be separated, even in the highest resolution spectrometers) has been confirmed for the first time in Del Zanna (1999). In normal quiet sun conditions, about $1 / 3$ of the intensity of the $335.4 \AA$ line is due to the Fe XII 335.339 A line.

Note that the Landi et al. (1997) calibration is in agreement with the sensitivity ratio imposed by these two lines, which constrain the relative sensitivity between these two wavelengths, with small error bars.

Fe XII (346.852, 352.106, $364.467 \AA)$

The brightest line, Fe XII $364.467 \AA$, is partly blended with the Si XI $364.497 \AA$ line only in active region spectra, not in the quiet sun observations used here. Fe XII $352.106 \AA$ is only slightly blended with an Al VII $352.149 \AA$ line (a Mg V $352.1 \AA$ does not contribute in the off-limb spectra), while the $346.852 \AA$ line is free of blends. These three Fe XII lines are all bright, free of blends in the off-limb quiet sun spectra, and the agreement between CHIANTI and SERTS data is excellent. These lines should therefore provide the best constraint for the CDS calibration in the central part of the NIS 1 detector. These lines indicate an almost constant sensitivity between $346.8 \AA$ and $364.5 \AA$ (the $352.106 \AA$ line was used to scale the sensitivity values of the lines shown in Fig. 7).

Fe XI $(341.113,352.662,356.519,369.153 \AA)$

The $369.153 / 352.662$ is a branching ratio, well represented in the SERTS-89 observations. These lines are bright and well resolved, in particular in off-limb spectra, and require a large increase in the sensitivity toward the higher wavelengths. The Fe XI $341.113 \AA$ is a bright well resolved line, that furthermore is well represented in the SERTS89 case (also noted by Young et al. 1998). However, in all the CDS spectra examined, this line is not well represented, and would require a much higher sensitivity than in the curve presented in Fig. 7 (the $352.662 \AA$ line was used as a reference line to scale the sensitivities). The Fe XI $341.113 \AA$ line therefore, must be blended in the CDS spectra. In the SERTS-89 spectrum, a very weak unidentified line is reported at $340.969 \AA$, with an intensity 0.17 times the intensity of the Fe XI line. Off-limb radial intensity profiles of the ratio of the $341.113 \AA$ with the other Fe XI lines (not shown here), although changing, do not present large variations, and therefore the unidentified line that blends the $341.113 \AA$ line must be a coronal line, and moreover not a high-temperature line (otherwise it would not contribute in the quiet sun on-disc or off-limb spectra). This therefore still leaves more than $30 \%$ of the observed line un-accounted for. This discrepancy would increase if a lower sensitivity at $341 \AA$ is adopted. Finally, the $356.519 \AA$ line is blended with an Fe XIV $356.641 \AA$ line and is not directly usable.

Fe X $(345.722,365.543 \AA)$

These two bright lines form a branching ratio. The $365.543 \AA$ line is strongly blended with a bright $\mathrm{Ne} \mathrm{V}$ line in on-disc observations. However, in off-limb observations, this line becomes free of blending, and can be used in conjunction with the $345.722 \AA$ to constrain the relative sensitivity between those wavelengths. This branching ratio would require a lower sensitivity at $345 \AA$ than is presented here, and is therefore not in agreement with the results obtained from the lines of Fe XII, unlike the SERTS-89 case (where, however, the $365.543 \AA$ line was strongly blended), nor with the requirements derived from the analysis of Fe XI. Considering the Fe XI results, the region between 340 and $350 \AA$ shows inconsistencies that remain unexplained (why should the same atomic data produce different results, if the SERTS-89 calibration was correct?), and will require more investigation.

Mg VIII (315.038, 335.253, 339.006 ^)

The Mg VIII $(315.038,339.006)$ lines always show a constant ratio (within a few \%), independently of the observed region and provide a good constraint on the relative sensitivity between these wavelengths. The $335.253 \AA$ line is only usable as a check, because even when the Fe XVI contribution is absent, there is a non-negligible contribution from an Fe XII line, as explained below.

Now, some more comments in Fig. 7 are needed. Although the Si IX and Si X lines are density-sensitive, and were therefore not used as calibration lines, their scaled sensitivities are also plotted in Fig. 7, to show that, within the errors, they are all consistent with the sensitivity curve that is presented here. If the $\mathrm{Fe} \mathrm{X}$ branching ratio is to be believed, then the sensitivity curve should be less constant in the $345-365 \AA$ range. This in turn would produce slightly lower densities as derived from the Si IX and the Si X ratios. However, this would leave unexplained why the Fe XI and Fe XII should be wrong. On the other hand, support to a constant sensitivity in the $340-355 \AA$ range comes from the preliminary CDS / SERTS-97 crosscalibration by Thomas et al. (1999).

\section{A.2. NIS 2}

\section{A.2.1. The transition region and chromospheric lines}

O III $(525.796,599.597 \AA)$

Note that in active region spectra the $525.796 \AA$ line is partly blended with Fe XVI $263 \AA$ (second order). There is also an O III line at $597.817 \AA$ which is weak and might be blended, although it is always in good agreement with the other two lines of the same ion. 
Table A.1. The ratios of the calibrated He I intensities of Vernazza \& Reeves (1978) for the various solar regions (QS: quiet sun; CH: coronal hole; AR: active region; cc: cell centres; net: network), with the theoretical ratios, calculated assuming optically thin emission and using the Arcetri Spectral Code. Note the small variations of these ratios, and how close they are to the predicted values. Below, the calibrated intensity of the $584.35 \AA$ is reported, to show the large variations of the observed intensities. At the bottom, the intensity ratio between the two resonance He I $584 \AA$ and He II $304 \AA$ lines is also shown. The coronal hole values have large uncertainties, and should be treated with caution, as noted by Vernazza \& Reeves (1978).

\begin{tabular}{llllllllll}
\hline ratio & QS avg & QS cc & QS net & CH avg & CH cc & CH net & AR & AR active calculated \\
\hline $515.62 / 584.35 \AA$ & 0.028 & 0.028 & 0.031 & 0.024 & 0.022 & 0.032 & 0.027 & 0.027 & 0.021 \\
$522.21 / 584.35 \AA$ & 0.050 & 0.048 & 0.053 & 0.054 & 0.054 & 0.055 & 0.043 & 0.045 & 0.044 \\
$537.03 / 584.35 \AA$ & 0.130 & 0.128 & 0.132 & 0.136 & 0.132 & 0.142 & 0.113 & 0.115 & 0.133 \\
\hline I (584.35 A) & 544.98 & 426.22 & 827.71 & 250.12 & 221.93 & 300.94 & 5470.68 & 7233.50 \\
\hline He I $(584) / \mathrm{He} \mathrm{II} \mathrm{(304)}$ & 0.065 & 0.057 & 0.075 & $0.105(?)$ & $0.114(?)$ & $0.082(?)$ & 0.075 & 0.079 \\
\hline
\end{tabular}

\section{O IV ( $553-556,608.397 \AA)$}

The O IV $608.397 \AA$ line was used in conjunction with any of the other O IV lines at 553-556 $\AA$. The O IV line (three transitions) visible at $617.0 \AA$ is very weak and partly blended with second order lines, and has not been used.

\section{He I $(515.62,522.21,537.03,584.35 \AA)$ and He II}

As noted by others (e.g. Andretta \& Jones 1997; Macpherson \& Jordan 1999), all the lines of the sequence present similar characteristics, independent of the type of solar region observed. In particular, the ratios of the line intensities versus the $584.35 \AA$ intensity is exceptionally constant (considering the large intensity variations), as shown in Table A.1, which reports the averaged (and calibrated) He intensities of Vernazza \& Reeves (1978). The same applies to the ratio of the He I/He II resonance lines, which is also constant, independent of the solar region observed (see Table A.1). These findings have been confirmed by the analysis of the NIS 2 spectra, performed for this calibration study. This fact makes these lines suitable for an in-flight calibration. Unfortunately, no accurate measurements of absolute-calibrated He I line intensities have been found in the literature, and the only set of well calibrated values are indeed those reported in Vernazza \& Reeves (1978), which in turn rely on previous rocket flight calibrations. However, assuming that the calibration is correct, then the CDS observations imply that the sensitivities at those wavelengths are those indicated by the large triangles in Fig. 9 (these have been scaled using the $584 \AA$ line as reference). The values that the sensitivities should have assuming optically thin He I emission (a questionable assumption for the He I lines, see Andretta \& Jones 1997; Macpherson \& Jordan 1999, and references therein), are also shown in the figure. It is interesting to note that: a) the sensitivity at $537 \AA$ is in excellent agreement with the value derived from the Vernazza \& Reeves (1978) calibrated values and the Brekke et al. (2000) value, but not with the ground calibration value; b) the 584 and $537 \AA$ lines appear to be effectively optically thin; c) the Vernazza \& Reeves (1978) intensities of the other two He I lines imply an upturn of the sensitivity at the shorter wavelengths, and not the downturn suggested by Brekke et al. (2000). However, the upturn required by the He I lines is not as steep as suggested by the Landi et al. (1997) study.

Regarding the He I/He II resonance lines ratios, all the NIS observations analysed have confirmed that this ratio is approximately constant, independent of the solar region observed, quite surprisingly. It is also interesting to note that the average value over quiet sun areas measured by CDS is about 0.07, in excellent agreement with the Skylab data, considering that the He II line was not separated from the Si XI $303 \AA$ resonance line.

\section{A.2.2. The coronal lines}

NIS 2 observes the bright doublets: Ca X (557.765, $574.010 \AA)$; $\mathrm{Al} \mathrm{XI}(550.031,568.12 \AA)$ and $\mathrm{Mg} \mathrm{X}(609.793$, $624.941 \AA$ ). In on-disc observations the Ca X $574.010 \AA$ is blended with a C III $574.279 \AA$ and the $\mathrm{Mg}$ X $609.793 \AA$ line is blended with a bright O IV $609.829 \AA$ line. The $\mathrm{Al}$ XI $568.12 \AA$ line is blended in active region observations with two second order lines (Fe XV and Al IX at 284.1 ), and in on-disc observations with Ne V 568.417 $\AA$. In particular, the two $\mathrm{Mg} \mathrm{X}$ lines have been used to determine the relative calibration between $610 \AA$ and $625 \AA$, using off-limb observations.

\section{A.3. NIS-GIS}

\section{A.3.1. Cross-calibration between the NIS 1 and NIS 2}

These lines that can be used are weak and partly blended. The lines observed in NIS 1 are:

1. a blend of Ne IV and Ne V at $357.8 \AA$. Some observations suggest that these lines are further blended; 
2. a weak and blended Ne V $359.382 \AA$ line;

3. a complex group of O III and N III transitions observed at $374 \AA$.

These lines were used as a cross-check of the relative NIS $1 /$ NIS 2 calibration in conjunction with the Ne IV (541.128, 542.072, 543.891 ^), Ne V (568.417, 569.824, $572.331 \AA$ ), and O III $(525.796,599.597 \AA)$ lines observed in NIS 2 (the N/O relative abundance can be checked using the N III and O III lines observed in GIS 4).

Only quiet sun on-disc observations, where the scattered light adds uncertainty to the measurements, can be used. Agreement with the results obtained from the other cross-calibrations, which used the GIS lines (described below), was found in most cases.

\section{A.3.2. Cross-calibration between NIS 1 and GIS 1}

Some of the bright lines that were used are:

- Fe X: 345.722, $365.543 \AA$ (NIS 1); 174.534, 177.242, 184.543, $190.0 \AA$ (GIS 1).

- Fe XI: 352.662, 356.519, $369.153 \AA$ (NIS 1); 180.4, $182.169,188.2(188.232+188.299) \AA$ (GIS 1).

- Fe XII: 346.852, 352.106, 364.467 A (NIS 1); 192.393 , 193.521, $195.100 \AA$ (GIS 1).

- Fe XIV: $334.172 \AA$ (NIS 1); $211.320 \AA$ (GIS 1).

\section{A.3.3. Cross-calibration between NIS 2, GIS 3, GIS 4}

The lines used for both the internal GIS 3 and GIS 4 calibration, and for the cross-calibration are (note that some of them are density-sensitive and were given lower importance in the iteration process):

- O III: 702.8 (2), 703.8 (2) Å (GIS 4); 525.796, 597.817 (bl Ca VIII), $599.597 \AA$ (NIS 2).

- N III: 763.334, 685.0, 685.8 (GIS 4).

- O V: 758.676, 759.441, 760.4 (2), 761.127, $762.003 \AA$ (GIS 4); $629.732 \AA$ (NIS 2).

- Ne IV: 469.9 (4) $\AA$ (GIS 3); 541.128 (bl Fe XIV IIo $270.51 \AA$ ), 542.072, 543.891 (bl Si X IIo $271.98 \AA$ ) $\AA$ (NIS 2).

- Ne V: 416.194, 481.3, 482.982 $\AA$ (GIS 3); 568.4 (bl), 569.8 (2), 572.3 (3) $\AA$ (NIS 2). The lines observed in GIS are of limited use, for various reasons.

- Ne VI: 399.821 (bl), 401.136 (bl), 401.926, $435.648 \AA$ (GIS 3); 558.594 (bl Ne VII), 562.803 (NIS 2).

- Ne VII: $465.22 \AA$ (GIS 3); 559.948, 561.7 (2), 564.528 (bl) $\AA$ (NIS 2).

- S IV 661.4 (2), 748.392, $753.759 \AA$ (GIS 4). Not that the $750.220 \AA$ is not in agreement with all the others.

\section{Appendix B: The comparison between observed and theoretical spectra}

A complete description of all the different spectra analysed can not preclude a detailed analysis of the identification of each line, including blends of all types (e.g., first with second order lines, lines with ghosts in the GIS). This is deferred to Paper II. Only a few types of spectra have been selected here, out of the large number of different spectra used for the calibration. In each case, the corresponding synthetic spectra are overlaid, with the addition of shaded areas under the curve. The second order contributions are also displayed, with darker shaded areas superimposed. The synthetic spectra have been created calculating the intensities of all the lines present in the CHIANTI database, and assuming a gaussian profile (except for the post-recovery case where broadened profiles were used). An indicative constant background (a good approximation in most cases) has been added to the synthetic spectra.

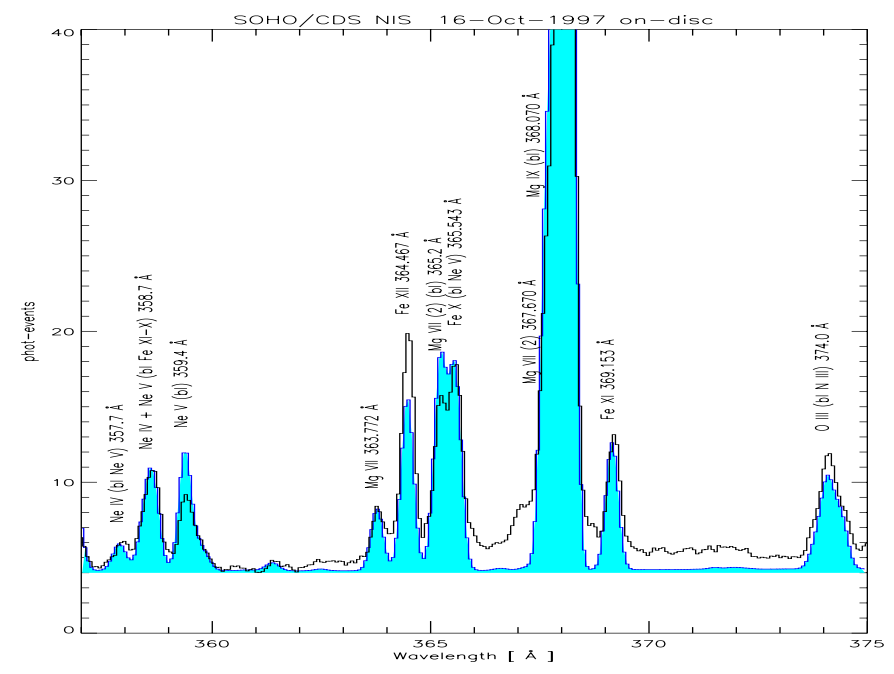

Fig. B.1. An on-disc observation of NIS 1, showing the few cool lines used for the cross-calibration between the two NIS channels in first order.

The figures in this Appendix are provided to give an overall view of some of the lines used for the calibration, and not necessarily to prove the agreement between observations and theory given by the calibration presented here. However, it is evident that the overall agreement is excellent, confirming the validity of the instrument calibration, together with the completeness of the CHIANTI atomic database. The main disagreements are either due to ghosting problems in the GIS or in consistent under- or overestimates of lines emitted by the same ion. For example, Fig. B.3 shows that both Al XI lines are over-estimated by the same amount, while the Si XI lines $(580.9 \AA$ and $303.3 \AA$ in second order) are consistently under-estimated. The figures in this Appendix also demonstrate the complexity of mixing between first and second order lines in the spectra, that becomes an important issue in all active region observations (see Del Zanna 1999 for details). 


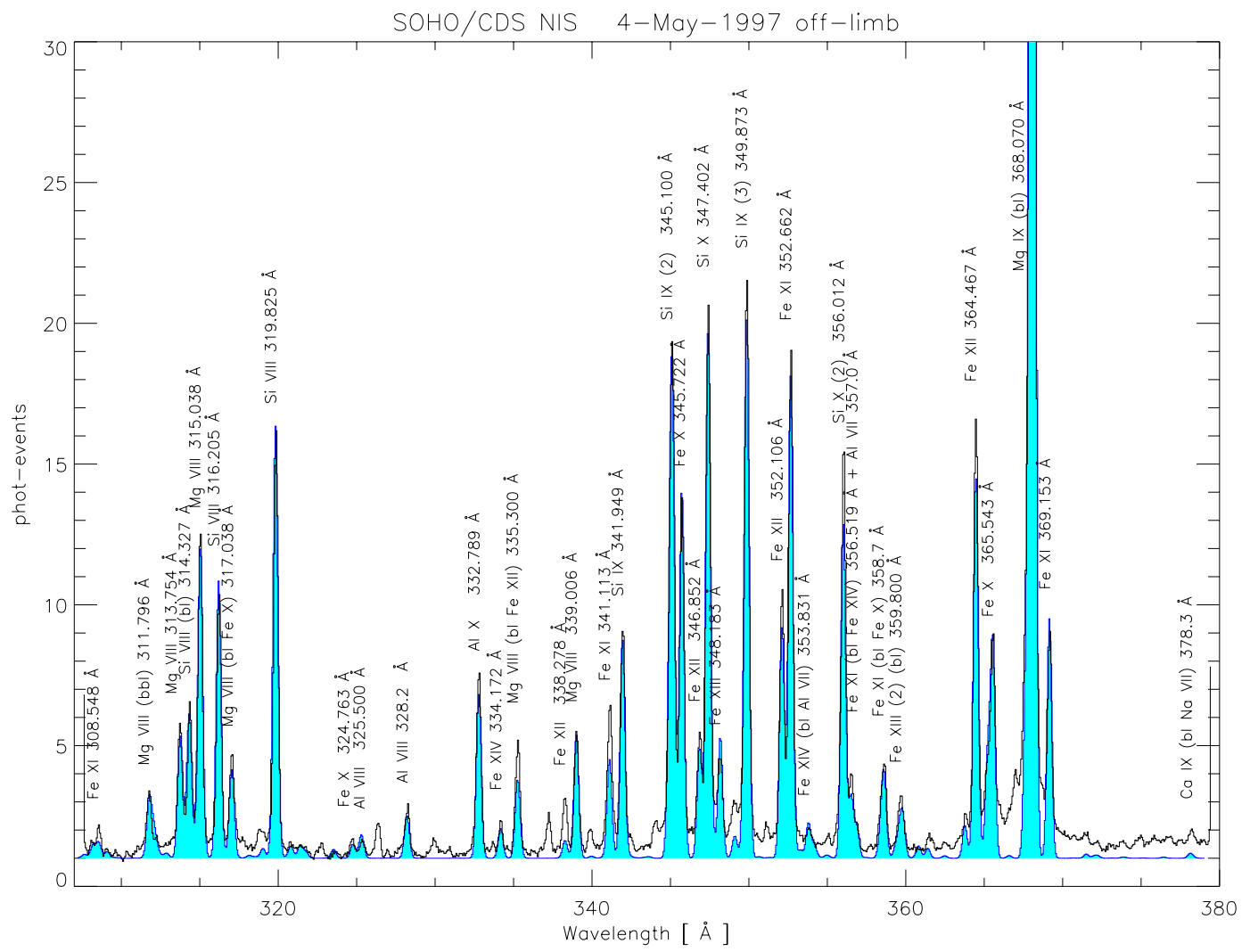

SOHO/CDS NIS 23-May-1999

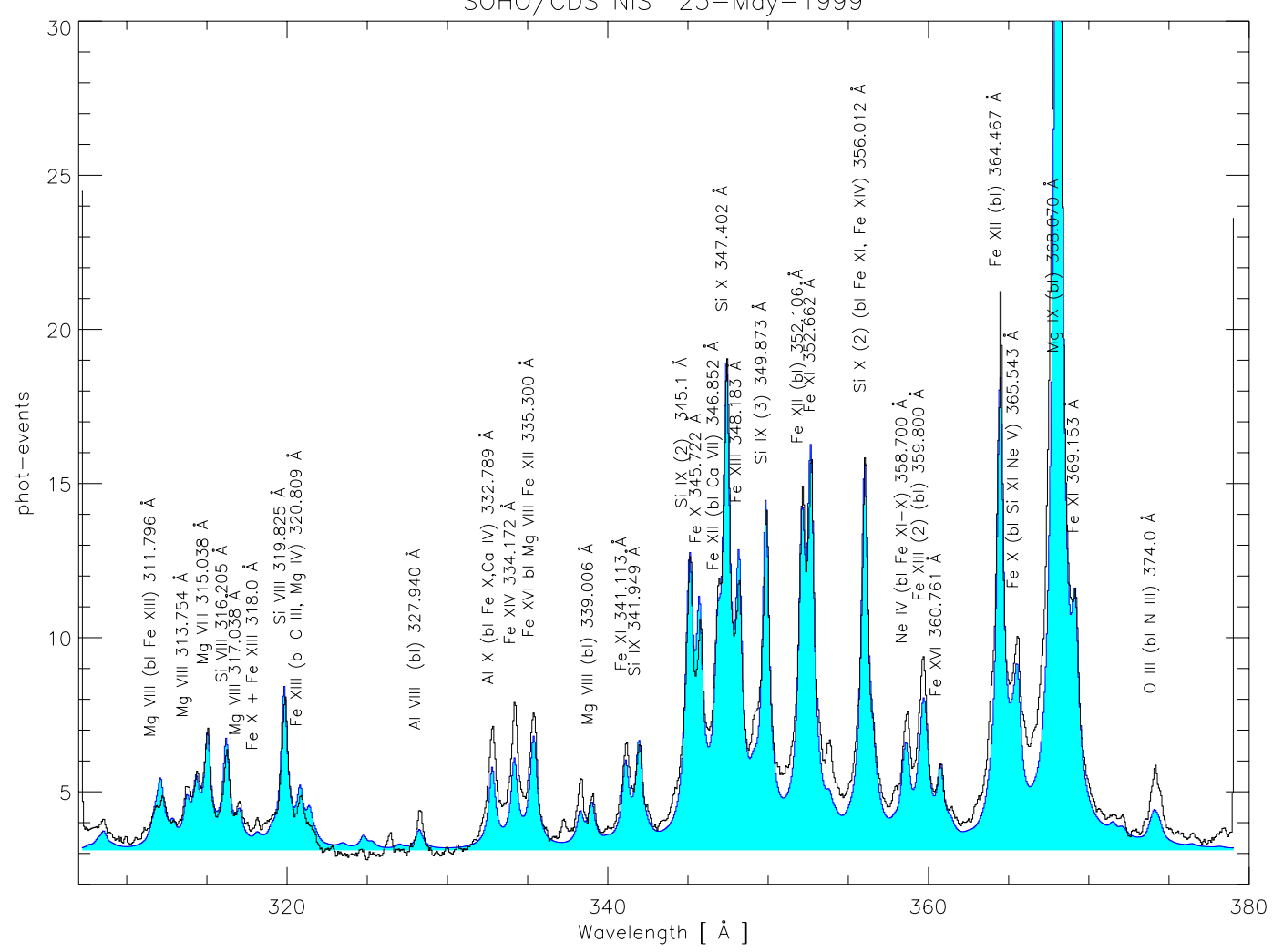

Fig. B.2. Top: the NIS 1 spectrum of an off-limb quiet sun observation (May 4th 1997), with corresponding synthetic spectrum overlaid. These types of spectra have been mainly used for the first order internal and cross-detector calibration. Bottom: an NIS 1 spectrum after SOHO loss. Note the strong blending of all lines, due to the broadened profiles. However, there are no obvious indications of a change of the calibration. 

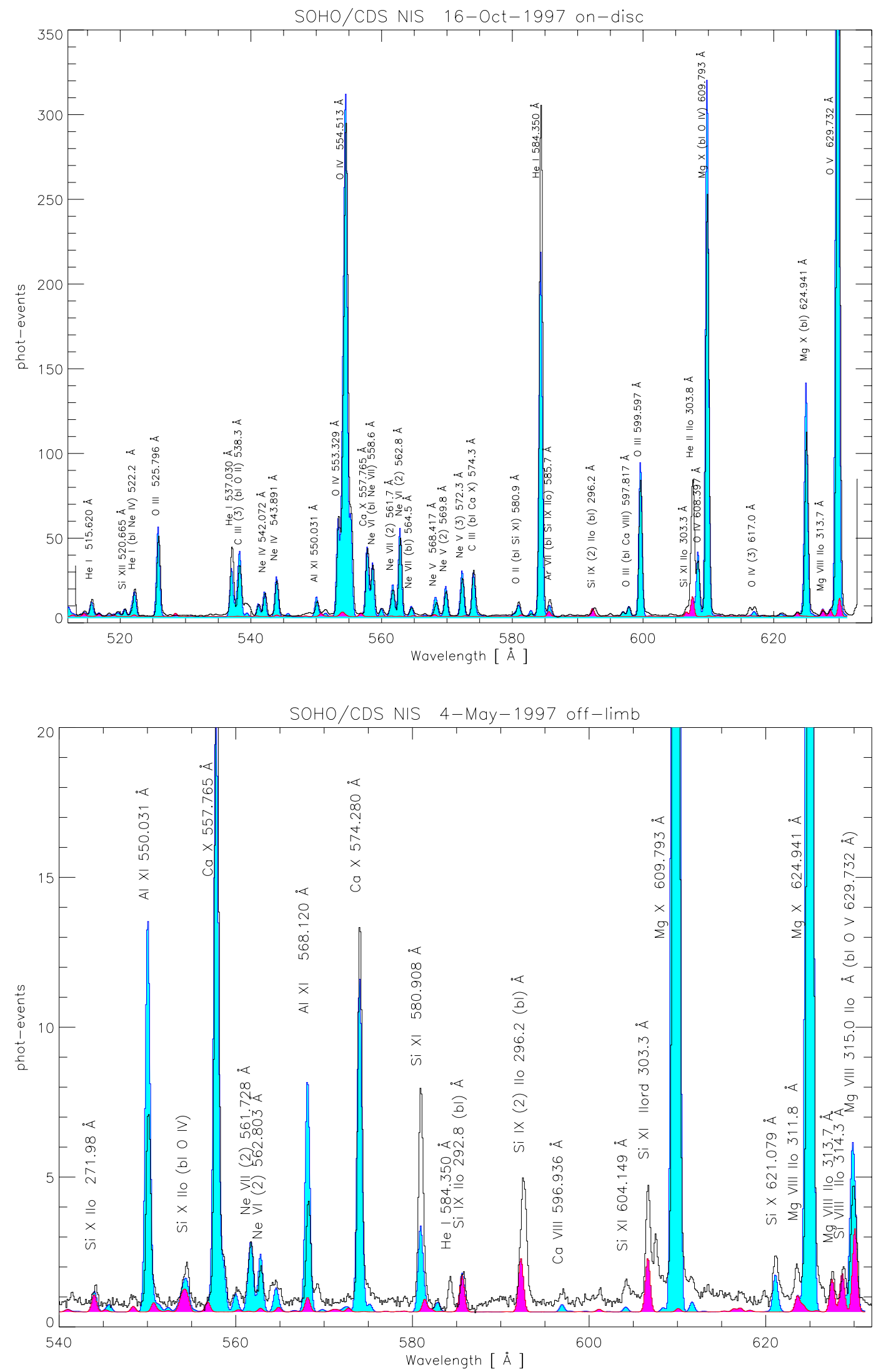

Fig. B.3. Top: the NIS 2 spectrum of an on-disc quiet sun observation. These types of spectra have been mainly used for the first order internal and cross-detector calibration using cool lines. Bottom: NIS 2 spectrum of an off-limb quiet sun observation. These types of observations have been used to constrain: a) the NIS 2 first order calibration using coronal lines (e.g. Al XI, $\mathrm{Ca} \mathrm{X}, \mathrm{Mg} \mathrm{X})$; b) the direct second order calibration, using the same lines observed in first order in the NIS 1 and GIS 2 channels. 

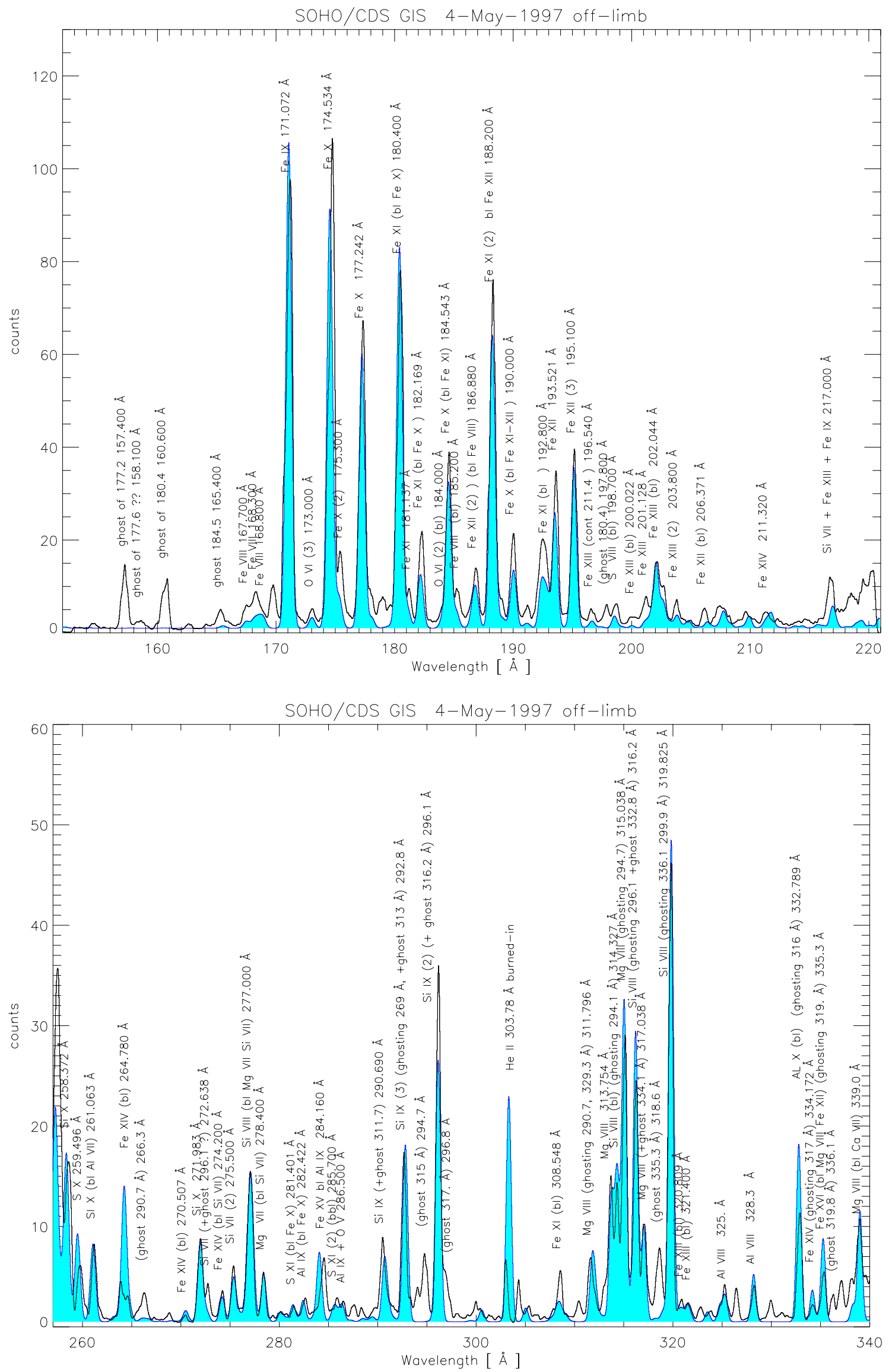

Fig. B.4. The GIS 1 and GIS 2 spectra of an off-limb quiet sun observation (May 4th 1997), with corresponding synthetic spectra overlaid. These types of spectra have been mainly used for the first order internal and cross-detector calibration. 

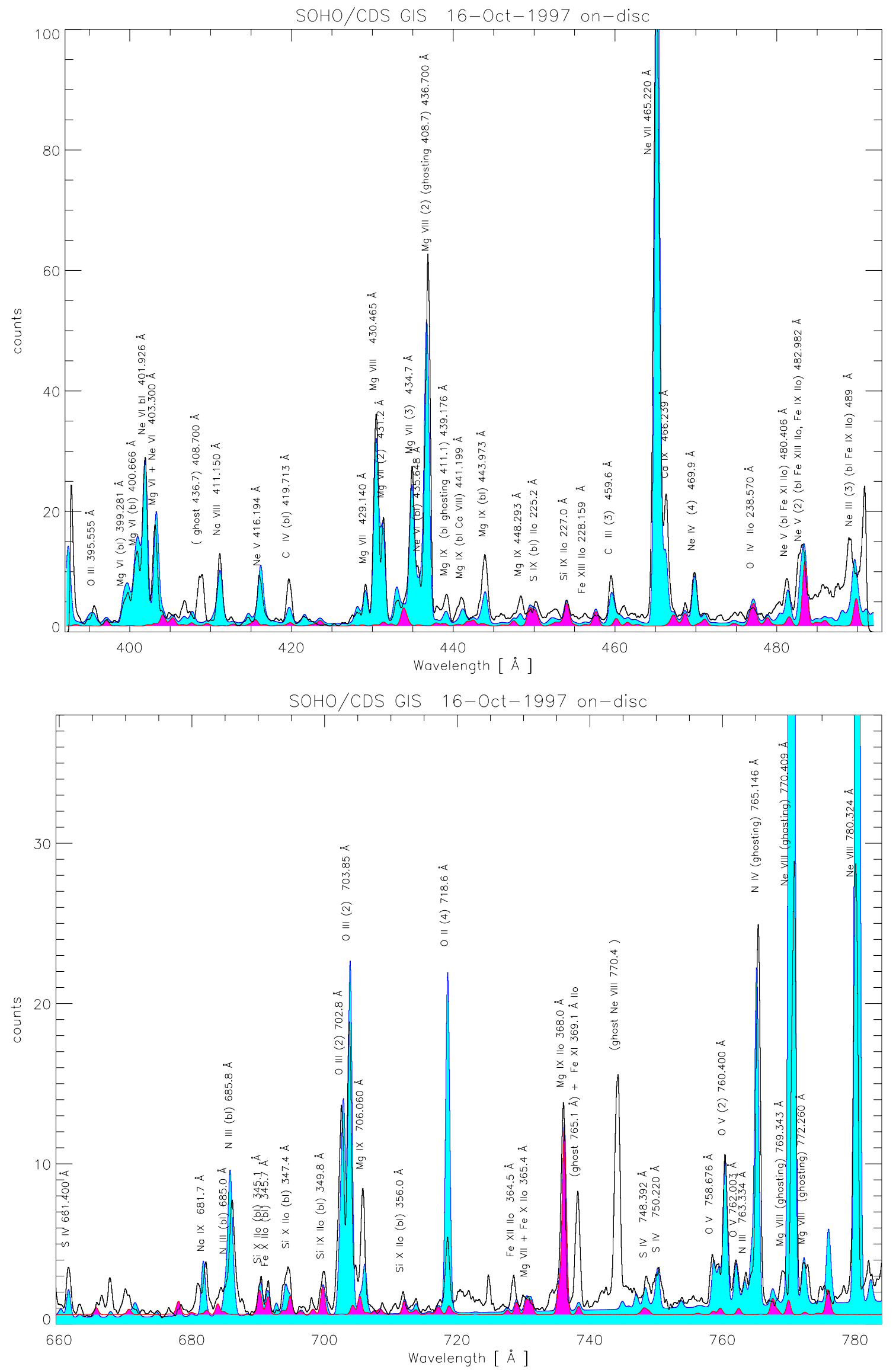

Fig. B.5. The GIS 3 and GIS 4 spectra of an on-disc quiet sun observation (October 16th 1997), with corresponding synthetic spectra overlaid. These types of spectra have been mainly used for the first order internal and cross-detector calibration. 


\section{References}

Allen, R., Landi, E., Landini, M., \& Bromage, G. E. 2000, A\&A, 358, 332

Andretta, V., \& Jones, H. P. 1997, ApJ, 489, 375

Arnaud, M., \& Rothenflug, R. 1985, A\&AS, 60, 425

Arnaud, M., \& Raymond, J. 1992, ApJ, 398, 394

Berrington, K. A., Burke, P. G., Dufton, P. L., \& Kingston, A. E. 1985, ADNDT, 33, 195

Binello, A. M., Landi, E., Mason, H. E., Storey, P. J., \& Brosius, J. W. 2001, A\&A, 370, 1071

Breeveld, A. A. 1996, Ph.D. Thesis, University of London

Brekke, P., Thompson, W. T., Woods, T. N., \& Eparvier, F. G. 2000, ApJ, in press

Brickhouse, N. S., Raymond, J. C., \& Smith, B. W. 1995, ApJS, 97,551

Bromage, B. J. I., Breeveld, A. A., Kent, B. J., Pike, C. D., \& Harrison, R. A., 1996, UCLan Report CFA/96/09

Brooks, D. H., Fischbacher, G. A., Fludra, A., et al. 1999, A\&A, 347, 277

Brosius, J. W., Davila, J. M., \& Thomas, R. J. 1998a, ApJ, 497, L113

Brosius, J. W., Davila, J. M., \& Thomas, R. J. 1998b, ApJS, 119,255

Craig, I. J. D. \& Brown, J. C. 1986, Inverse Problems in Astronomy (Adam Hilger, Bristol)

Del Zanna, G., \& Bromage, B. J. I. 1999a, J. Geophys. Res., 104, 9753

Del Zanna, G., \& Bromage, B. J. I. 1999b, ESA SP-446, 269

Del Zanna, G. 1999, Ph.D. Thesis, University of Central Lancashire

Del Zanna, G., Bromage, G. E., Landini, M., \& Mason, H. E. 2001 , in preparation

Dere, K. P., Landi, E., Mason, H. E., Monsignori Fossi, B. C., \& Young, P. R. 1997, A\&AS, 125, 149

Dere, K. P., Landi, E., Young, P. R., \& Del Zanna, G. 2001, ApJS, 134, 331

Doschek, G. A., Mariska, J. T., \& Feldman, U. 1981, MNRAS, 195,107

Doschek, G. A., Laming, J. M., Feldman, U., et al. 1998b, ApJ, 504,573

Dupree, A. K. 1972, ApJ, 178, 527

Gianetti, D., Landi, E., \& Landini, L. 1999, ESA SP-448, 599

Grevesse, N., \& Anders, E. 1991, Solar element abundances, in Solar Interior and Atmosphere, ed. A. N. Cox, W. C. Livingston, \& M. S. Matthews (Tucson: Univ. Arizona Press), 1227
Harrison, R. A., Sawyer, E. C., Carter, M. K., et al. 1995, Sol. Phys., 162, 233

Haugan, S. V. H. 1997, CDS software, note No. 47

Haugan, S. V. H. 1999, Sol. Phys., 185, 275

Judge, P. G., Woods, T. N., Brekke, P., \& Rottman, G. J. 1995, ApJ, 455, L85

Jupen, C., Isler, R. C., \& Trabert, E. 1993, MNRAS, 264, 627

Landi, E., Landini, M., Pike, C. D., \& Mason, H. E. 1997, Sol. Phys., 175, 553

Landi, E., \& Landini, M. 1998b, A\&A, 340, 265

Landi, E., \& Landini, M. 1998a, A\&AS, 133, 411

Landi, E., Del Zanna, G., Breeveld, E. R., et al. 1999a, A\&AS, 135,171

Landi, E., Landini, M., Dere, K. P., Young, P. R., \& Mason, H. E. 1999b, A\&A, 135, 339

Lang, J., Kent, B. J., Breeveld, A. A., et al. 1999, RAL Technical Report RAL-TR-1999-036

Lang, J., Kent, B. J., Breeveld, A. A., et al. 2000, J. Opt. A, $2,88-106$

Macpherson, K. P., \& Jordan, C. 1999, MNRAS, 308, 510

Mason, H. E., Young, P. R., Pike, C. D., et al. 1997, Sol. Phys., 170,143

Mason, H. E., Landi, E., Pike C. D., \& Young, P. R. 1999, Sol. Phys., 189, 129

Mazzotta, P., Mazzitelli, G., \& Colafrancesco 1998, A\&AS, 133, 403

McIntosh, S. W. 2000, ApJ, 533, 1043

Monsignori Fossi, B. C., \& Landini M. 1991, Adv. Space Res., 11,281

Neupert, W. M., \& Kastner, S. O. 1983, A\&A, 128, 181

Pauluhn, A., Rüedi, I., Solanki, S. K., et al. 1999, App. Opt., 38,7035

Storey, P. J., Mason, H. E., \& Young, P. R. 2000, A\&AS, 141, 285

Thomas, R. J., \& Neupert, W. M. 1994, ApJS, 91, 461

Thomas, R. J., Davila, J. M., Thompson, W. T., Kent, B. J., \& Hollandt, J. 1999, BAAS, 31, 850

Thompson, W. T. 1999, priv. comm.

Vernazza, J. E., \& Reeves, E. M. 1978, ApJS, 37, 485

Warren, H. P., Mariska, J. T., \& Lean, J. 1998, J. Geophys. Res., 103, 12077

Young, P. R., \& Mason, H. E. 1997, Sol. Phys., 175, 523

Young, P. R., Landi, E., \& Thomas, R. J. 1998, A\&A, 329, 291

Zhang, H. L., \& Sampson, D. H. 1992, ADNDT, 52, 143 